\title{
Massive Stars in the Tarantula Nebula: A Rosetta Stone for Extragalactic Supergiant HII Regions
}

\author{
Paul A. Crowther ${ }^{\mathbb{D}}$ \\ Department of Physics \& Astronomy, University of Sheffield, Sheffield S3 7RH, UK; \\ paul.crowther@sheffield.ac.uk
}

Received: 3 September 2019; Accepted: 6 November 2019; Published: 8 November 2019

\begin{abstract}
A review of the properties of the Tarantula Nebula (30 Doradus) in the Large Magellanic Cloud is presented, primarily from the perspective of its massive star content. The proximity of the Tarantula and its accessibility to $X$-ray through radio observations permit it to serve as a Rosetta Stone amongst extragalactic supergiant HII regions since one can consider both its integrated characteristics and the individual properties of individual massive stars. Recent surveys of its high mass stellar content, notably the VLT FLAMES Tarantula Survey (VFTS), are reviewed, together with VLT/MUSE observations of the central ionizing region NGC 2070 and HST/STIS spectroscopy of the young dense cluster R136, provide a near complete Hertzsprung-Russell diagram of the region, and cumulative ionizing output. Several high mass binaries are highlighted, some of which have been identified from a recent X-ray survey. Brief comparisons with the stellar content of giant HII regions in the Milky Way (NGC 3372) and Small Magellanic Cloud (NGC 346) are also made, together with Green Pea galaxies and star forming knots in high-z galaxies. Finally, the prospect of studying massive stars in metal poor galaxies is evaluated.
\end{abstract}

Keywords: galaxies; star formation-galaxies; star clusters; general-open clusters and associations; individual; 30 Doradus-stars; massive-stars; early-type

\section{Introduction}

The Tarantula Nebula (alias 30 Doradus) in the Large Magellanic Cloud (LMC) is the brightest supergiant HII region in the Local Group of galaxies, and serves as a local analogue to metal-poor starburst knots in high redshift galaxies [1]. Its proximity $(50 \mathrm{kpc}$ ), and high galactic latitude (and hence low extinction) have permitted a myriad of ground-based and space-based surveys across the electromagnetic spectrum, revealing an exceptional population of massive stars $\left(\geq 8 M_{\odot}\right)$, including the dense, young star cluster R136 that is home to some of the most massive stars known. The advent of modern highly multiplexed spectrographs coupled with large ground-based telescopes, has permitted multi-epoch optical spectroscopic surveys of the massive star population of the Tarantula Nebula for the first time. The VLT FLAMES Tarantula Survey, hereafter VFTS [2], has provided the multiplicity, rotational velocities and initial mass function of massive stars. In distant starburst regions it is not possible to resolve individual stars, such that studies rely on techniques based on their integrated properties, which themselves involve assumptions of binarity, stellar rotation and mass function.

The Tarantula Nebula is not the sole example of a supergiant HII region within the Local Group. However, studies of the richest Milky Way star-forming regions are limited by dust extinction (e.g., Westerlund 1, NGC 3603). Counterparts in other Local Group galaxies suffer from a number of limitations, including a relatively modest stellar content (NGC 346 in the Small Magellanic Cloud, $\mathrm{SMC}$ ) or much greater distance (NGC 604 in M33), such that the Tarantula-whose metallicity is approximately half-solar [3]—serves as the only credible Rosetta Stone for rich extragalactic star-forming regions. This review will provide a brief overview of the structural properties of 
the Tarantula Nebula, but will largely focus on its massive star content, drawn from results from VFTS and other optical spectroscopic surveys, supplemented by the recent deep Chandra X-ray survey 'Tarantula-Revealed by X-rays' (T-ReX). Finally, comparisons with local and high-redshift star-forming regions will be provided to put the properties of the Tarantula into a broader context. Indeed, the nebular properties of the central NGC 2070 region of the Tarantula are strikingly similar to Green Pea galaxies, exhibiting intense [O III] $\lambda 4959,5007$ emission, and its star-formation rate is comparable to intense star-forming clumps at high redshift.

\section{Tarantula Nebula}

The Tarantula Nebula is the most striking star-forming region in the LMC, whether viewed in the far ultraviolet (hot, luminous stars), $\mathrm{H} \alpha$ (ionized gas) or mid-IR (warm dust), extending over several hundred parsec, owing to a massive stellar content producing an ionizing output which is a thousand-fold higher than the Orion Nebula [4].

NGC 2070 is the dominant ionized region within the Tarantula Nebula, powered by a large number of hot luminous stars from R136 at its heart plus many more within its vicinity. NGC 2060, located $6 \operatorname{arcmin}(90 \mathrm{pc})$ to the southwest, is host to a more modest population of OB stars plus an X-ray pulsar PSR J0537-6910 and its supernova remnant (SNR) N157B. Hodge 301 is located 3 $\operatorname{arcmin}(45 \mathrm{pc})$ to the north west of R136, but does not possess significant nebulosity since previous supernovae are likely to have cleared this region of gas, and its stellar population (B-type stars and red supergiants) does not possess a significant Lyman continuum output. NGC 2070 is often referred to as a cluster in the literature but it extends over tens of parsecs whereas genuine star clusters are an order of magnitude smaller, such that the only rich star clusters within the Tarantula are R136, with an age of 1-2 Myr [5] and Hodge 301 with an age of 20-30 Myr [6] with a few additional lower mass young, compact clusters (e.g., TLD1, SL 639). Table 1 compares various regions within the Tarantula Nebula, adapted from a previous review by Walborn [7]. Although the focus of the present review is on spectroscopic results for massive stars, Sabbi et al. [8] have undertaken a deep Hubble Space Telescope (HST) multi-colour photometric survey, known as the Hubble Tarantula Treasury Project (HTTP) which permits lower mass stars in the Tarantula Nebula to be studied.

Table 1. Physical scales within the Tarantula Nebula, adapted from Walborn [7]. Ionizing outputs, $\mathrm{N}(\mathrm{LyC})$ are obtained from the present work.

\begin{tabular}{|c|c|c|c|c|c|}
\hline $\operatorname{Region}_{\mathbf{R}}$ & $\begin{array}{c}\text { Angular } \\
\text { Radius (") }\end{array}$ & $\begin{array}{c}\text { Physical } \\
\text { Radius (pc) }\end{array}$ & $\begin{array}{c}\mathrm{N}(\mathrm{LyC}) \\
\left(10^{51} \mathrm{ph} \mathrm{s}^{-1}\right)\end{array}$ & Content & Reference \\
\hline R136a & 0.8 & 0.2 & 2 & R136a1 (WN5h), R136a2 (WN5h) & [9] \\
\hline R136 & 4 & 1.0 & 4 & R136b (O4 If/WN8), R136c (WN5h+) & [5] \\
\hline NGC 2070 & 080 & 20. & 9 & R140a (WC4 + WN6+), Mk34 (WN5h + WN5h) & {$[10,11]$} \\
\hline Tarantula & 600 & 150. & 12 & Hodge 301, PSR J0537-6910 (pulsar), N157B (SNR) & [12] \\
\hline
\end{tabular}

A number of complementary studies of the star formation history of the Tarantula Nebula have been carried out, exploiting pre-main sequence low mass stars [13,14] and massive stars [15]. Significant star formation commenced $\sim 25 \mathrm{Myr}$ ago, as witnessed by Hodge 301 , and reached a peak several Myr ago, with the young massive star cluster R136 at its heart no more than 2 Myr old. It is apparent that star formation within the Tarantula Nebula has not been limited to specific parsec-scale star clusters, such as Hodge 301 or R136, but has been distributed across the entire region, akin to a super OB association. Wright et al. [16] have established from proper motion observations that star formation in the far smaller Milky Way Cygnus OB2 region did not originate in a star cluster, but involved individual sub-regions in virial equilibrium. Indeed, median ages of massive stars show little radial dependence on their projected distance from R136, with very massive stars $\left(\geq 100 M_{\odot}\right)$ identified throughout the region [15]. Infrared and radio observations of the Tarantula reveal ongoing regions of massive star formation, to which the reader is referred to the review by Walborn [17] and a more recent study of the brightest embedded sources based on Spitzer/IRAC imaging [18]. Atacama 
Large Millimetre Array (ALMA) has obtained high resolution observations of parsec-scale clumps within the Tarantula [19], with the rate of star formation in the Tarantula anticipated to decline in the future.

\section{Massive Star Content}

The integrated nebular properties of the Tarantula Nebula and other selected giant $\mathrm{H}$ II regions in the Local Group is presented in Table 2. The ionizing output of the Tarantula Nebula corresponds to the equivalent of over a thousand O7V stars, each with $10^{49} \mathrm{ph} \mathrm{s}^{-1}$. In reality, the Tarantula hosts somewhat fewer O-type stars since the most extreme examples-early $\mathrm{O}$ stars and luminous Wolf-Rayet (WR) stars-each produce an order of magnitude more Lyman continuum photons. Nevertheless, this population represents an order of magnitude more $\mathrm{O}$ and WR stars than any Milky Way or SMC giant HII regions, and is not likely to be improved upon until extremely large telescopes are capable of resolving the massive stellar content of more extreme giant HII regions, such as NGC 5461, 5462 and 5471 in M 101 [1].

Table 2. Integrated nebular properties of nearby giant HII regions, adapted from Kennicutt [20] for an assumed O7V Lyman continuum output of $10^{49} \mathrm{ph} \mathrm{s}^{-1}$.

\begin{tabular}{lccr}
\hline Region (Galaxy) & $\begin{array}{c}\text { Diameter } \\
\text { pc }\end{array}$ & $\begin{array}{c}\mathbf{L}(\mathbf{H} \alpha) \\
\mathbf{1 0}^{\mathbf{3 9}} \mathbf{\mathbf { e r g ~ s } ^ { - \mathbf { 1 } }}\end{array}$ & N(O7V) \\
\hline NGC 3372 (Milky Way) & 200 & 0.8 & 45 \\
NGC 346 (SMC) & 220 & 0.8 & 45 \\
NGC 3603 (Milky Way) & 100 & 1.5 & 110 \\
NGC 604 (M33) & 400 & 4.5 & 320 \\
Tarantula (LMC) & 370 & 15 & 1100 \\
\hline
\end{tabular}

Historically, there have been several photometric and spectroscopic surveys of early-type stars in the Tarantula Nebula, each of which have employed contemporary (Galactic) temperature calibrations to produce Hertzsprung-Russell diagrams. Parker [21,22] obtained the first extensive study of the entire region. Subsequently, Massey and Hunter [23] obtained high-spatial resolution HST spectroscopy of early-type stars in the central, crowded R136 region, revealing an exceptional population of very early O stars, whilst Melnick and colleagues $[24,25]$ obtained spectroscopy for a large number of early-type stars in the NGC 2070 region. The advent of efficient multi-object spectrographs on 8-10 $\mathrm{m}$ telescopes, has permitted the most comprehensive optical spectroscopic survey of massive stars in the Tarantula Nebula to date through the VLT FLAMES Tarantula Survey (VFTS) [2]. Multi-object, multi-epoch spectroscopy of $\sim 800$ massive stars across the entire region, for which detailed spectroscopic analyses have been undertaken for over $500 \mathrm{O}$ and early B stars, such that temperature calibrations are no longer necessary. Although this represents the most extensive of early-type stars in a single star-forming region to date, this survey is incomplete owing the fibre-placement limitations, sampling $\sim 70 \%$ of massive stars exterior to the dense R136 cluster from comparison with photometric surveys [12].

Two additional surveys have recently provided complete optical spectroscopic observations of all bright sources within the central crowded region of the Tarantula: (a) the central $4 \operatorname{arcsec}(1 \mathrm{pc}$ ) of the R136 cluster exploiting the high spatial resolution of HST/STIS [5]; (b) the central $2 \times 2$ arcmin $(30 \times 30$ pc) region of NGC 2070 using the VLT/MUSE integral field spectrograph [10]. Although these lack the multi-epoch capabilities of VFTS, they are complementary since the richest stellar populations of the Tarantula are found within NGC 2070/R136, and provide extended wavelength coverage (yellow and red for MUSE, ultraviolet for STIS), albeit at reduced spectral resolution. A summary of these three contemporary surveys is provided in Table 3, together with literature results. In total, approximately 1100 early-type massive stars have been spectroscopically observed. Our discussion of the massive star content of the Tarantula will largely draw upon results from VFTS, but include others where appropriate. It should be noted that analyses based on spectroscopic fibre-fed observations of early-type stars in regions of strong, highly variable nebulosity, is inherently problematic owing 
to the lack of local sky subtraction. Such issues do no affect long-slit STIS or integral field MUSE observations.

Table 3. Summary of stellar content of the Tarantula Nebula from recent spectroscopic surveys (excluding sources in common, although including individual components within SB2 binaries).

\begin{tabular}{llcccccc}
\hline Telescope/inst & Target & N(O-type) & N(B-type) & N(WR) & N(Of/WN) & N(A+) & Reference \\
\hline VLT /FLAMES & 30 Dor & 369 & 436 & 9 & 6 & 35 & {$[2]$} \\
HST/STIS & R136 & 57 &.. & 3 & 2 &.. & {$[5]$} \\
VLT/MUSE & NGC 2070 & 115 & 79 &.. &.. & 1 & {$[10]$} \\
Other & 30 Dor & 29 & 8 & 16 &.. & 5 & {$[22-24,26]$} \\
\hline Total & 30 Dor & 570 & 523 & 28 & 8 & 41 & \\
\hline
\end{tabular}

VFTS and other surveys have revealed that the Tarantula hosts extreme examples of stellar exotica, including the most massive stars known [9], a Luminous Blue Variable, R143 [27], a very massive runaway [28], the fastest rotating stars [29], a massive overcontact binary [30], and a supernova remnant N157B [31], with SN 1987A located $~ 300$ pc to the south west (Figure 1).

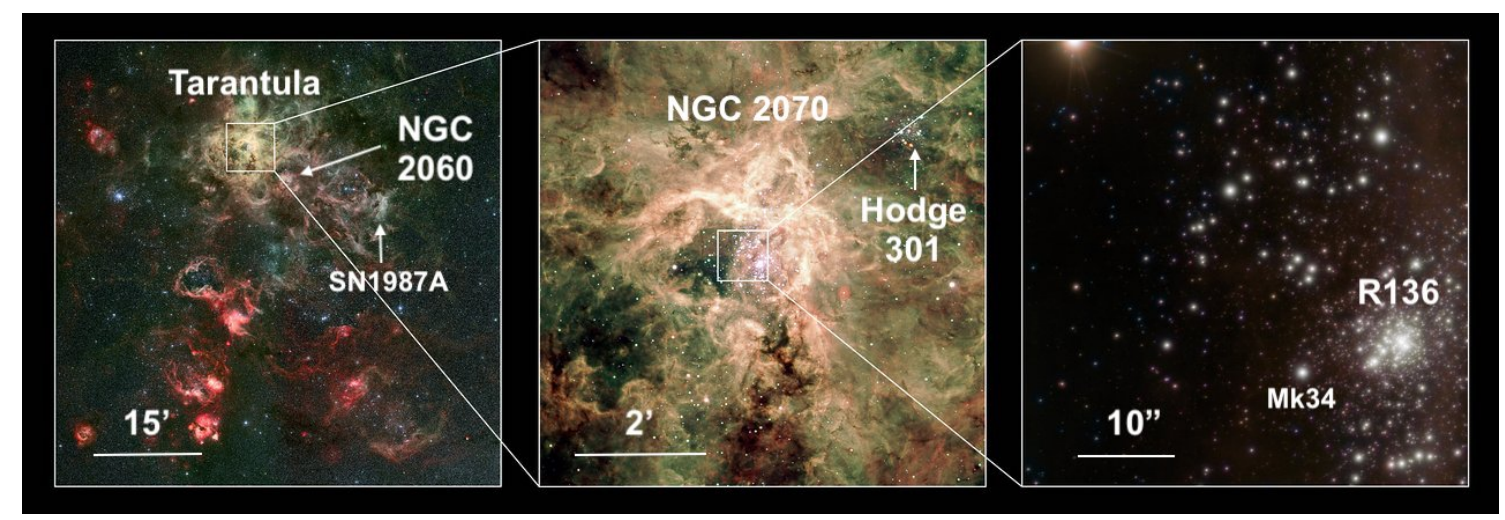

Figure 1. (left) Optical image of the Tarantula Nebula from the MPG/ESO 2.2m WFI, with NGC 2060 and SN1987A indicated; (centre) Optical VLT/FORS2 image centred on NGC 2070, with Hodge 301 to the upper right; (right) an infrared VLT/MAD image of the central R136 region, with the massive colliding wind binary Mk 34 indicated. Credit: ESO/P. Crowther/C.J. Evans.

R136a, the central cluster, merits special consideration since it was considered by some to be a supermassive star as recently as the early 1980s [32]. Speckle interferometry resolved R136a into multiple sources [33], and it was subsequently established as a compact star cluster [34]. Massey and Hunter [23] established that dozens of the brightest sources within the central parsec were hot, early O stars. Spectroscopic studies of the brightest components R136a1, a2, a3, with nitrogen-sequence Wolf-Rayet spectral types, indicated masses of $\sim 100 M_{\odot}$ [23,35]. They established that these relatively weak-lined WN stars are luminous main-sequence stars close to their Eddington limits, rather than classical Wolf-Rayet stars. Subsequent analyses of the WN stars in R136 indicated significantly higher masses of 150-300 $M_{\odot}$ [9] as a result of increased spectroscopic luminosities, owing to higher stellar temperatures and IR photometry less affected by dust extinction. Indirectly inferred masses of massive stars are notoriously imprecise, and if binarity were established for individual stars their inferred luminosities and masses would be reduced. To date, faint companions to members of R136a have been detected with extreme adaptive optics imaging [36]. Melnick 34 is spectroscopically similar to the WN5-stars in R136a, and has recently been shown to be a colliding wind binary system comprising two WN5 components, with a total mass exceeding $250 M_{\odot}$ [37]. Figure 2 shows that Melnick 34 is an order of magnitude brighter than R136a in X-rays, indicating that there are no colliding wind binaries comparable to Melnick 34 within R136a [38]. 

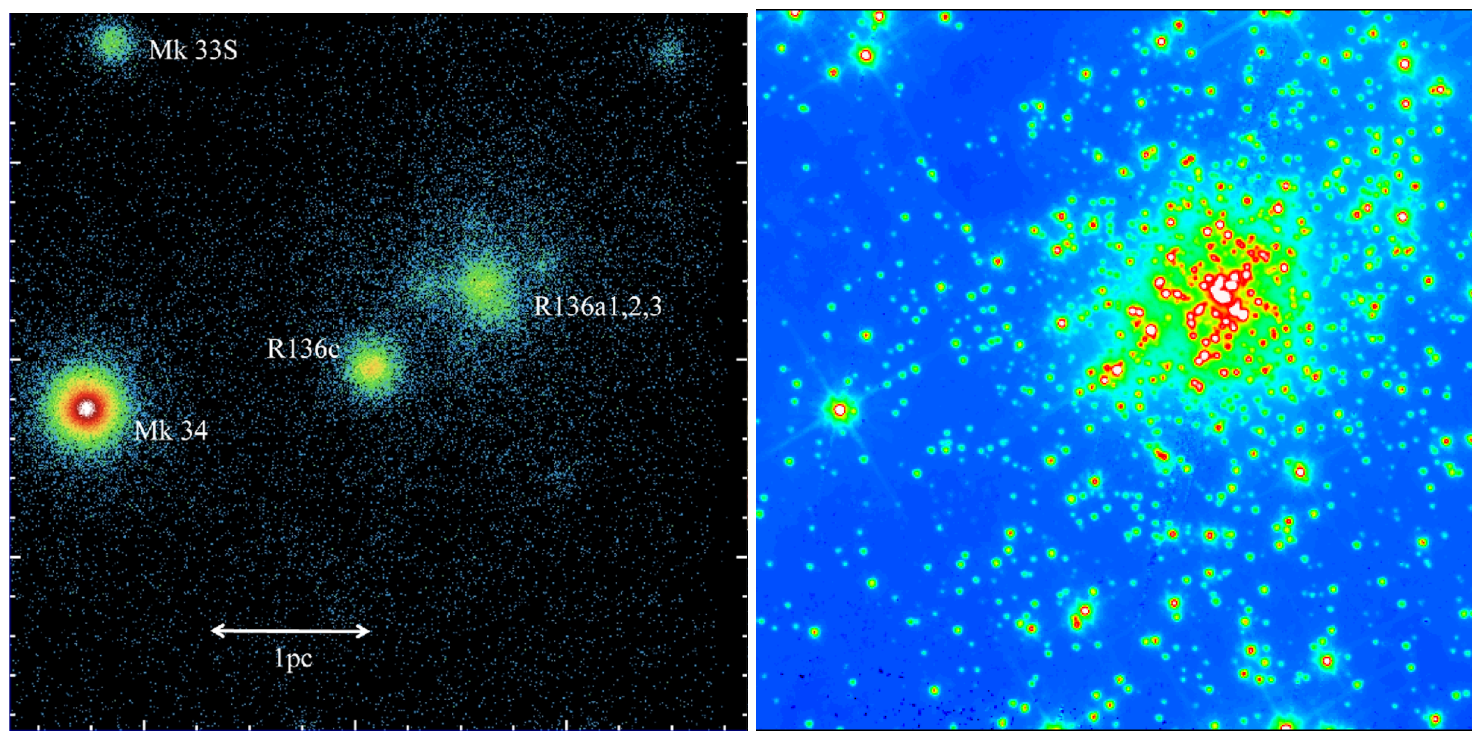

Figure 2. (left) Chandra ACIS X-ray logarithmic intensity image of the core of NGC 2070 from T-ReX, centred on R136c, adapted from [38], showing the relative brightness of the colliding wind binary Melnick 34 (WNh5 + WN5h) [37] to the R136a star cluster (hosting multiple WN5h stars) and R136c (WN5h+?); (right) HST WFC3/F555W logarithmic intensity image of the same $19 \times 19$ arcsec region, highlighting the rich stellar population of R136a with respect to R136c and Melnick 34.

Overall, the Tarantula hosts a remarkable number of $\sim 50$ early-type stars with bolometric luminosities exceeding $10^{6} \mathrm{~L} \odot$. For reference, the Milky Way's Carina Nebula (NGC 3372) hosts $\approx 5$ massive stars with such extreme properties [39]. As such, the Tarantula Nebula represents our best opportunity to study the highest mass stars known, both individually and collectively. Schneider et al. [40] analysed VFTS spectroscopic results to establish an excess of massive stars with respect to a standard Salpeter Initial Mass Function ( I MF), indicating 1/3 more stars with $\geq 30 M_{\odot}$ in 30 Doradus compared to expectations from a standard IMF. Finally, although we focus primarily on high mass early-type stars in the Tarantula Nebula, it also hosts red supergiants (RSG). Since RSG are the evolved descendants of moderately massive stars, and star formation in the Tarantula has peaked relatively recently, of order $\sim 10$ RSG are known, most of which are associated with mature star clusters Hodge 301 and SL 639 [6].

\section{Physical Properties}

The determination of physical properties $\left(T_{\mathrm{eff}}, \log g\right)$ of individual early-type stars ideally requires high S/N $(\geq 50)$ intermediate resolution spectroscopy of suitable diagnostics, usually He I-II lines (Si II-IV) for temperatures of O-type (B-type) stars, plus Balmer lines for surface gravities, plus grids of model atmospheres obtained with modern codes, such as FASTWIND [41] for O stars and blue supergiants, CMFGEN [42] or PoWR [43] for emission-line stars, or TLUSTY [44] for low luminosity B stars. Analysis of late-type supergiants requires model atmosphere codes in which molecular opacities have been incorporated, such as MARCS [45]. Hot $\mathrm{O}$ stars require alternative temperature diagnostics to helium, with nitrogen commonly used since the blue visual spectrum of $\mathrm{O}$ stars includes lines of N III-V. Wolf-Rayet stars are especially problematic since photospheres are masked by the dense wind, such that gravities cannot be directly measured and temperatures usually refer to deep layers, with an optical depth of $\tau \sim 10-20$, rather than the effective temperature at $\tau=2 / 3$. If stellar distances are uncertain, comparisons with evolutionary models can be made using the so-called spectroscopic Hertzsprung-Russell (sHR) diagram [46], involving temperature and $\mathscr{L}=T_{\text {eff }}^{4} / g$, the inverse of the flux-weighted gravity, where $g$ is the surface gravity.

The determination of stellar luminosities requires comparisons between synthetic spectral energy distributions and photometry, taking account of interstellar extinctions and distance moduli, 18.5 mag 
in the case of the Large Magellanic Cloud. Visual extinctions of early-type stars in the Tarantula Nebula are usually modest, although near-IR photometric comparisons usually lead to more robust luminosities since typical dust extinctions are $0.1-0.2 \mathrm{mag}$ in the K-band, versus 1-2 mag in the $\mathrm{V}$-band, and the lack of sensitivity of $\mathrm{K}$-band extinctions to any variations in the overall extinction law. Luminosities of RSG can also be reliably estimated by integrating observed spectral energy distributions from visual to mid-IR wavelengths [47,48]. Historically, stellar estimates of masses and ages from evolutionary models involved by-eye comparisons between their position on a conventional Hertszprung-Russell (HR) diagram and theoretical isochrones. However, additional physical information is often available, such as helium abundance or projected rotational velocities. Tools now exist which additionally take such information into account for the calculation of stellar ages and initial masses such as BONNSAI [49]. Significant discrepancies exist between current mass estimates from spectroscopic ( $\log g$ ) and evolutionary approaches for a subset of VFTS O dwarfs [50].

Figure 3 presents the HR diagram of the Tarantula Nebula, comprising single star results from VFTS [50-54], VLT/MUSE [11], HST/STIS [55] and literature results for other stars within 160 parsec of R136, including Wolf-Rayet stars [56]. Results for binary systems have been incorporated, primarily drawn from [57] for VFTS B-type binaries, Tarantula Massive Binary Monitoring (TMBM) for VFTS O-type binaries [58] and recent literature for WR stars [37,59]. Evolutionary tracks for non-rotating, LMC metallicity massive stars up to the onset of He-burning have been included for reference [60,61]. Over 1170 massive stars have been included, revealing a well populated main sequence population up to $\sim 200 M_{\odot}$, plus classical Wolf-Rayet stars to the left of the main sequence, and evolved blue supergiants up to $\log \left(L / L_{\odot}\right) \sim 6$, and cool supergiants, up to $\log \left(L / L_{\odot}\right) \sim 5.3$ [48]. The addition of all luminous early-type stars from R136 and NGC 2070 fills in the extreme upper main sequence which is somewhat under populated from VFTS alone [40]. The overwhelming majority of the older massive stellar population-i.e., evolved stars with masses below $30 M_{\odot}$-are spatially exterior to NGC 2070 (open symbols), although NGC 2070 is host to one luminous M supergiant, Melnick 9. Conversely, beyond NGC 2070, the main-sequence population at the highest stellar masses is relatively underpopulated, albeit with several WN5h stars (R146, R147) and early O stars (VFTS 16, BI 253) located $95 \pm 25$ parsec from R136.

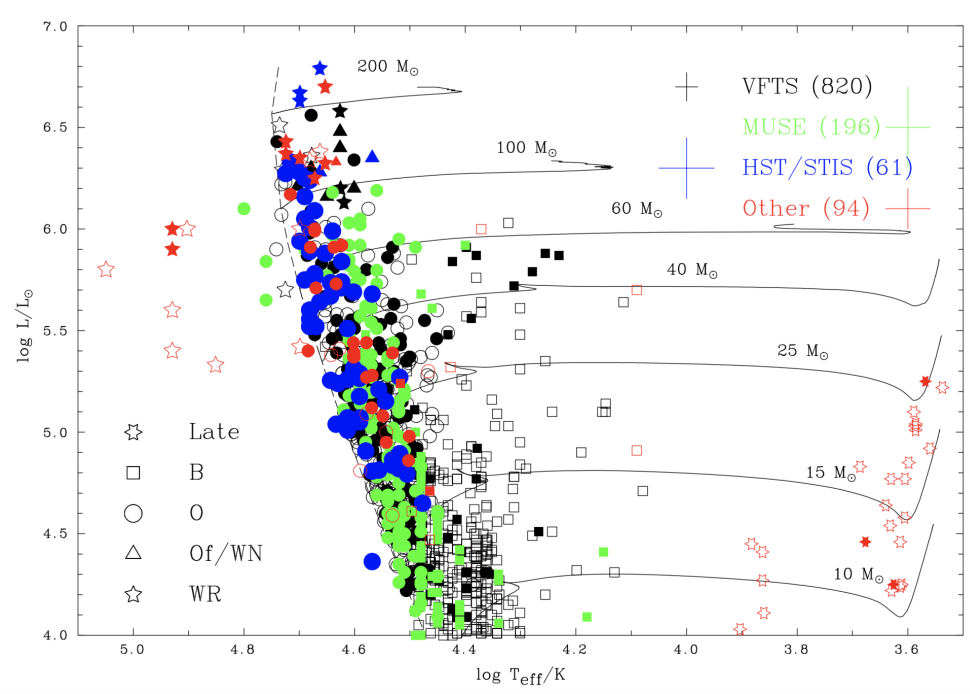

Figure 3. Hertzsprung-Russell diagram of the Tarantula Nebula, based on results from VLT FLAMES Tarantula Survey (VFTS) [50-54], MUSE [11], Hubble Space Telescope (HST)/STIS [55] and other literature results, with typical uncertainties from each survey indicated. Filled symbols are within NGC 2070, open symbols elsewhere in the Tarantula. Non-rotating tracks for 10, 15, 25, 40, 60, 100 and $200 M_{\odot}$ LMC metallicity stars have been included from [60,61] which terminate at the onset of He-burning. 


\section{Binaries, Rotation and Runaways}

Until recently, the significance of close binary evolution for massive stars was not fully recognised, in spite of a few binary "champions" [62]. The high frequency of close binaries amongst O stars in young Galactic clusters obtained from radial velocity monitoring established that only a minority of massive stars follow single stellar evolution [63]. In contrast with the majority of previous spectroscopic surveys of early-type stars, VFTS comprised multiple epochs, such that [64] were able to establish that $53 \%$ of $\mathrm{O}$ stars in the Tarantula Nebula inhabit a binary system with a period below 1500 days, such that binary interaction will occur. In total, $18 \%$ of O-type binaries, those with very short periods, are anticipated to merge with a companion, $27 \%$ will be stripped of their envelopes (primaries, mass donors) and $8 \%$ are predicted to be spun up (secondaries, mass gainers), as summarised in Figure 4 together with counterparts in Milky Way clusters. Broadly similar results have been obtained for VFTS B-type stars [65].

The inferred rate of envelope stripping and spin-up in the Tarantula is rather lower than [63] obtained for O stars in Milky Way clusters (Figure 4), but it is probable that the true incidence is rather higher since a subset of the current $\mathrm{O}$ star sample is likely to have already undergone binary evolution. Although VFTS has established the binary frequency amongst massive stars in the Tarantula, binary orbits require follow-up surveys, notably the TMBM survey [58,66].

Consequences of close binary evolution include mass gaining secondaries being spun-up, and a subset of secondaries possessing high space velocities as a result of the disruption of the binary following the core-collapse supernova (ccSN) of the original primary. Other "observables" are more challenging, including the identification of stripped primaries in close binaries which will usually be masked at visual wavelengths by mass gaining secondaries [67] which should be more common in older star clusters such as Hodge 301.
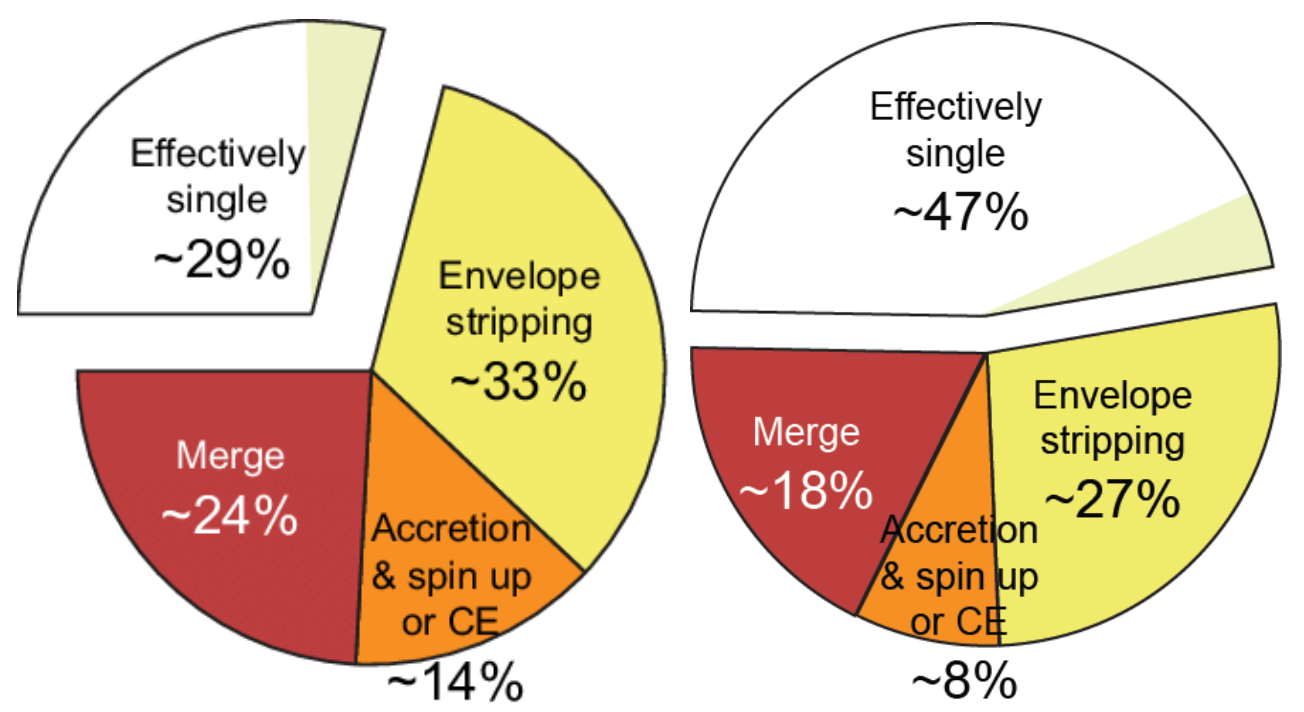

Figure 4. Pie charts, courtesy Hugues Sana and Selma de Mink, illustrating the fraction of O stars undergoing single stellar evolution versus mergers, primaries being stripped of their envelopes, and secondaries being spun up, for Milky Way young clusters (left) and VFTS O stars (right), adapted from $[63,64]$.

Reliable measurements of projected rotation rates, $v_{e} \sin i$, are often problematic because strong hydrogen and helium lines are predominantly affected by pressure broadening, with an additional contribution from "macroturbulence". A Fourier Transform approach applied to metallic lines offers the most robust results, albeit requiring high resolution, high $\mathrm{S} / \mathrm{N}$ spectroscopy of suitable diagnostics [68]. The lack of a spectral features originating in the hydrostatic layers of Wolf-Rayet stars prevents a direct determination of their rotational velocities. Figure 5 compares projected rotational velocities for a 
large sample of VFTS $\mathrm{O}$ and B-type stars. Typical rotational velocities of single $\mathrm{O}$ stars are modest, with $v_{e} \sin i \sim 100 \mathrm{~km} \mathrm{~s}^{-1}$, albeit with $10 \%$ exceeding $300 \mathrm{~km} \mathrm{~s}^{-1}$ [69], of which some examples are rotating close to their critical rates [29]. This high velocity tail is suspected of being spun-up mass gainers in former close binaries. The lack of fast rotators amongst O stars in VFTS binary systems supports this interpretation [70]. Figure 5 illustrates that rotational velocities of VFTS B-type stars are higher, with $v_{e} \sin i \sim 200 \mathrm{~km} \mathrm{~s}^{-1}$ on average, and $20 \%$ exceeding $300 \mathrm{~km} \mathrm{~s}^{-1}$ [71].

Of particular interest is the rotational velocity distribution of massive stars within the young R136 star cluster, whose severe crowding prevented inclusion in VFTS. Bestenlehner et al. [55] utilised HST/STIS spectroscopy to reveal $150 \mathrm{~km} \mathrm{~s}^{-1}$ on average for a sample of 55 massive stars within the central parsec of R136, with no examples exceeding $250 \mathrm{~km} \mathrm{~s}^{-1}$, although the low $\mathrm{S} / \mathrm{N}$ of these datasets prevented distinguishing between rotational and macroturbulence, adding to the interpretation that rapid rotators originate from close binary evolution. Wolff et al. [72] obtained somewhat higher rotational velocities for $\mathrm{OB}$ stars in the periphery of R136, where contamination from the field population is significant.

The Tarantula hosts a number of candidate early-type runaway stars from their measured (radial and /or tangential) velocities with respect to the average for their environment, although radial velocity outliers may be unresolved binaries. Runaways can originate either from disrupted secondaries following the core collapse of primaries in close binaries, or following the dynamical ejection of stars from young star-forming regions. Platais et al. [73] have investigated high proper motion stars in the Tarantula from HST imaging obtained 3 years apart, revealing a number of potential stars ejected from R136, while Lennon et al. [28] have exploited Gaia DR2 proper motions to conclude that VFTS 16 (O2 III) [74] was likely ejected from R136 during its formation 1-2 Myr ago. Renzo et al. [75] discuss the origin of the candidate 'walkaway' very massive star VFTS 682 (WN5h).

The Tarantula hosts several notable massive binary systems, whose physical and orbital properties have been obtained from spectroscopic monitoring, with searches for massive binaries also greatly benefitting from the recent Chandra T-ReX survey (PI Leisa Townsley) which monitored the Tarantula in X-rays for almost 2 years with a total integration time of $2 \mathrm{Ms}$. Single hot, luminous stars tend to produce (thermal) $\mathrm{X}$-rays due to shocks in the winds, but these are generally soft $\mathrm{X}$-ray emitters with $L_{X} / L_{\mathrm{Bol}} \sim 10^{-7}$ [76]. Massive stars in binary systems may lead to excess $X$-ray emission arising from wind-wind collisions, usually relatively hard, providing the separations are not too small (low wind velocities) or too large (low wind densities) [77]. A close binary comprising an early-type star and compact remnant (neutron star or black hole) will be extremely X-ray bright if the accretion disk of the remnant is being fed by the wind of the massive star or via Roche Lobe overflow.

A number of eclipsing binaries in the proximity of R136 have been identified [78], including \# 38 from [34] comprising an $\mathrm{O} 3 \mathrm{~V}+\mathrm{O} 6 \mathrm{~V}$ in a circular 3.4 day orbit, with component masses 57 and $23 M_{\odot}$. This represented the first robust stellar mass determination for an O3 star in the LMC. VFTS revealed a large number of binaries within the Tarantula, many of which have been followed-up with TMBM. Most notably R139 has been established as an eccentric system comprising a pair of mid O supergiants in a 154 day orbit [79] with lower limits of $\sim 66+78 M_{\odot}$ for individual component, recently revised downward to $54+69 M_{\odot}$ [58]. R139 is amongst the brightest X-ray sources in the Tarantula in T-ReX with $L_{\mathrm{X}, \text { corr }} \sim 5 \times 10^{33} \mathrm{erg} \mathrm{s}^{-1}$ and an enhanced $L_{\mathrm{X} \text {,corr }} / L_{\mathrm{Bol}} \sim 9 \times 10^{-7}$ based on TMBM bolometric luminosities [58]. 


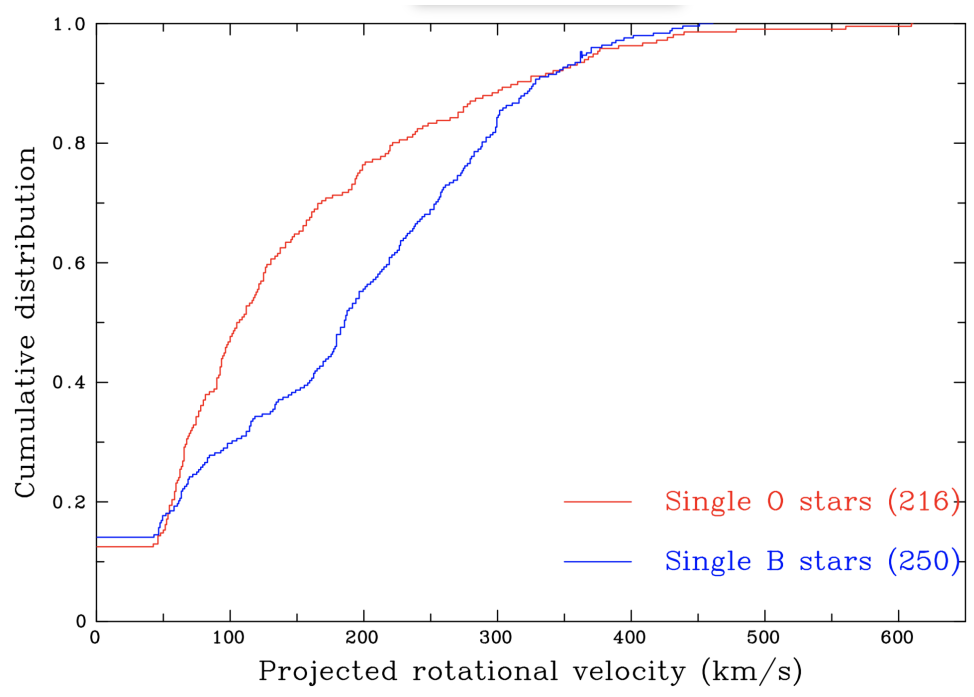

Figure 5. Cumulative distribution of rotational rates for single VFTS O (red) and B stars (blue) [69,71].

The most remarkable X-ray source in the Tarantula is VFTS 399 with $L_{X, c o r r} \sim 5 \times 10^{34} \mathrm{erg} \mathrm{s}^{-1}$ despite being associated with a low luminosity O9 giant, implying $L_{\mathrm{X}, \text { corr }} / L_{\mathrm{Bol}} \sim 2 \times 10^{-4}$. Clark et al. [80] conclude that VFTS 399 is a high-mass X-ray binary hosting a neutron star remnant, with the $\mathrm{O}$ giant known to be a rapid rotator $\left(v_{e} \sin i=324 \mathrm{~km} \mathrm{~s}^{-1}\right.$, according to [53]), as one would expect for a mass gainer in a close binary system.

Two point sources in the Tarantula are even brighter in X-rays than VFTS 399. Of these, R140a is a compact group of stars including two WR stars, so likely hosts one or more colliding wind binaries, while X-ray variability for Melnick 34 reveals a 155 day period, peaking at $L_{X, c o r r}=3.2 \times 10^{35} \mathrm{erg} \mathrm{s}^{-1}$, exceeding $\eta$ Carina at X-ray maximum [38]. Melnick 34 has been confirmed to be a colliding wind binary system in a 155 day eccentric orbit, with minimum masses of 60-65 $M_{\odot}$ for each of the WN5h components [37]. Individual masses of 130-140 $M_{\odot}$ are favoured from spectroscopic analysis, such that Melnick 34 is likely to be the most massive binary known to date, with $L_{\mathrm{X}, \mathrm{corr}} / L_{\mathrm{bol}}=1.7 \times 10^{-5}$ at $\mathrm{X}$-ray maximum. This arises from the collision of dense, fast moving winds at a minimum separation of $\sim 1.2 \mathrm{AU}$ or 13 stellar radii for an assumed orbital inclination of $i=50^{\circ}$. Almeida et al. [30] identify the overcontact binary VFTS 352 as a prototype of systems which, at low metallicity, are plausible black hole-black hole merger progenitors.

\section{Wind Properties}

The underlying theory responsible for outflows by hot luminous stars has been known for several decades [81], with a metallicity dependence primarily arising from the variation in iron-peak elemental abundances $[82,83]$. Individual wind properties of $\mathrm{O}$ stars or blue supergiants usually rely on spectroscopic fits to $\mathrm{H} \alpha$, as parameterised by the wind strength parameter, $Q$ [41], from which the mass-loss rate requires knowledge of the physical radius (from $T_{\text {eff }}, \log L$ ) and measured or adopted wind velocity. Wind velocities of $\mathrm{OB}$ stars cannot be measured from optical spectroscopy, so usually spectral type calibrations are adopted based on measured velocities from UV P Cygni profiles of C IV, $\mathrm{NV}$ or Si IV [84]. Until recently, high S/N, high quality UV spectroscopy of OB stars in the Large Magellanic Cloud has been in short supply, but the situation has improved via the HST Large Program METAL (GO 14675, PI Julia Roman-Duval) [85] and upcoming ULLYSES initiative ${ }^{1}$.

1 http://www.stsci.edu/stsci-research/research-topics-and-programs/ullyses. 
Specifically for the Tarantula, low resolution UV spectroscopy of the R136 star cluster has added a significant number of wind measurements for early $\mathrm{O}$ stars [5]. Wind velocities exceed $3000 \mathrm{~km} \mathrm{~s}^{-1}$ at the earliest subtypes (O2-3), reducing to $\sim 1500 \mathrm{~km} \mathrm{~s}^{-1}$ for late O-types. Mass-loss rates of emission line stars typically rely on an alternative wind scaling relation, namely the transformed radius, $R_{t}$ [86], which also requires knowledge of wind velocities, although these can be estimated from optical spectroscopy for Wolf-Rayet stars. An added complication arises because radiatively-driven winds are known to be inherently unstable, leading to clumped winds. Wolf-Rayet winds have been known to be clumped for 30 years [87,88], but the degree of clumping for O stars via the $\mathrm{H} \alpha$ diagnostic remains unclear, with conflicting results obtained from UV resonance lines [89] unless the wind comprises a mixture of optically thin and thick clumps within a much lower density inter-clump medium [90].

Figure 6 presents unclumped mass-loss rates of $\mathrm{O}$, Of/WN and Wolf-Rayet stars in the Tarantula Nebula, obtained from VFTS [50,51,53], HST/STIS [55] and other literature results. Uncertainties have been included wherever possible. Since the primary wind diagnostic in the majority of instances presented here is $\mathrm{H} \alpha$, it is apparent that uncertainties are large for those stars with weak stellar winds. In addition, mass-loss rates for Of/WN and Wolf-Rayet stars are anticipated to be reduced significantly owing to wind clumping, as indicated with downward arrows. If volume filling factors are $\sim 10 \%$, mass-loss rates will be reduced by a factor of $\sqrt{10}$.

Theoretical mass-loss rates [83] for zero-age main sequence stars at LMC composition [60,61] are included in Figure 6. At face value it would appear that the theoretical mass-loss rates of LMC O stars are supported by theory. However, the following should be borne in mind. It is not clear how significantly wind clumping affects the inferred mass-loss rates of normal $\mathrm{O}$ stars, although $\mathrm{H} \alpha$ results for supergiants are likely to be sensitive to wind clumping. In addition, the vast majority of mass-loss rates of VFTS O stars shown here have been inferred by adopting wind velocities from an assumed scaling relation involving escape velocities [91,92], which are themselves dependent upon spectroscopic gravities. Exceptions are HST/STIS results for early-type stars in R136 which are based on measured UV wind velocities, and span dwarfs, giants, supergiants and main sequence WN stars. In order to verify predictions for lower luminosity $(\log L / L \odot<5.5)$ O stars, more sensitive diagnostics would need to be employed, such as UV P Cygni lines, providing complications such as porosity are accounted for [90].

It is clear from Figure 6 that rates for the highest luminosity main-sequence Of/WN and WN stars significantly exceed theoretical predictions. This discrepancy is partially addressed through wind clumping, but very massive stars close to their Eddington limits are observed to exhibit enhanced mass-loss rates which are not taken into account in standard theoretical predictions [93,94]. Unsurprisingly, classical Wolf-Rayet stars with $\log \left(L / L_{\odot}\right)=5.5-6$ possess the strongest winds amongst early-type stars in 30 Doradus, with clumping-corrected wind densities an order of magnitude higher than O stars with similar luminosities. It is well known that the wind momenta of WR stars, $\dot{M} v_{\infty}$, exceeds the momentum provided by their radiation field, $L / c$, owing to multiple photon absorption and re-emission within their optically thick winds, permitting $\dot{M} v_{\infty} /(L / c)>1$ [95]. 


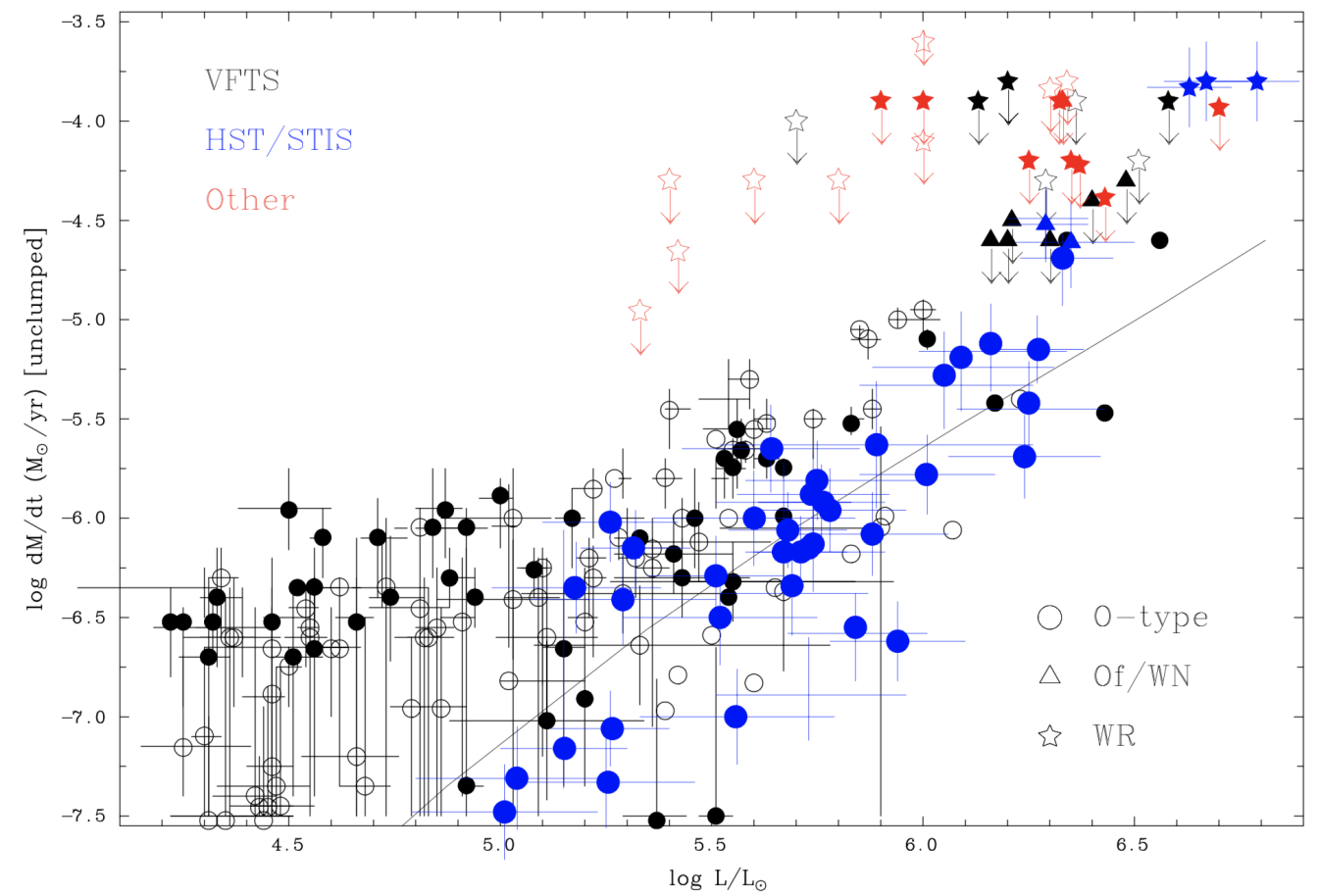

Figure 6. Unclumped mass-loss rates of O-type, Of/WN and Wolf-Rayet stars in the Tarantula Nebula (based on results from VFTS [50,51,53], HST/STIS [55] and other surveys [37,56,59] for WR stars). Filled symbols are within NGC 2070, open symbols elsewhere in the Tarantula. Theoretical mass-loss rates for zero age main sequence massive stars at the Large Magellanic Cloud (LMC) metallicity [83] are included (solid line), based on LMC metallicity evolutionary models $[60,61]$.

\section{Fate of Massive Stars in the Tarantula}

The conventional picture of massive star evolution close to solar metallicity is that those with initial masses of 8-25 $M_{\odot}$ will end their lives as red supergiants (RSG), undergo a H-rich core collapse supernova, leaving behind a neutron star remnant, while higher mass counterparts will either circumvent the RSG phase or subsequently proceed to a Wolf-Rayet stage prior to undergoing core collapse, leading to a H-deficient supernova (neutron star remnant) or faint/failed supernova (black hole remnant) [96]. If one considers the global WR vs. RSG population in the LMC, the lower boundary to the luminosity of WR stars is $\log \left(L / L_{\odot}\right)=5.3$, while the upper luminosity of RSG is $\log (L / L \odot)=5.5$ [48], supporting a transition from RSG to WR for higher mass progenitors at $\sim 25 M_{\odot}$.

Close binary evolution severely complicates this scenario, since primaries below $25 M_{\odot}$ can be stripped of their hydrogen envelope, leading to a type $\mathrm{Ilb}$ or $\mathrm{Ib} / \mathrm{c}$ instead of a H-rich supernova, while secondaries will be rejuvenated, spun-up, with the potential for a core-collapse supernova for secondaries whose initial masses fall below $8 M_{\odot}$. To date, there are no unambiguous cases of pre-supernova close binaries in the Tarantula hosting stripped (Wolf-Rayet or helium) stars, although it has been suggested that the WN3 binary BAT99-49 elsewhere in the LMC is the product of close binary evolution [59]. Rapid rotation of the bright $\mathrm{O}$ giant component of the high mass X-ray binary VFTS 399 is consistent with this evolutionary scenario. The absence of low luminosity Wolf-Rayet stars in the Tarantula does not exclude the binary channel since low-mass stripped stars would be unlikely to exhibit a Wolf-Rayet spectral appearance [67].

Initially very close binaries may merge on the main sequence, prior to following a relatively conventional evolution, albeit with unusually high rotation rates, which would lead to increased luminosities and potentially evolve blueward off the main sequence $[60,61]$. Extremely rapid rotation in some VFTS OB stars favours close binary evolution or stellar mergers. Very massive stars in the Tarantula up to $\sim 300 M_{\odot}$ are expected to lead to $30-50 M_{\odot}$ CO cores and black hole fates, unlikely to produce any associated supernova [97], such that a subset of binary VMS are plausible progenitors 
of LIGO black hole binary mergers, although their exact fate crucially depends on their mass-loss properties, which remain uncertain.

\section{Integrated Properties and Comparison With Star-Forming Regions, Near And Far}

The Tarantula Nebula would subtend little more than one arcsec if it were located at a distance of $50 \mathrm{Mpc}$, and so provides us with a unique opportunity to compare the individual spatially-resolved properties of an intensively star-forming region and its aggregate characteristics. Using integrated $\mathrm{H} \alpha$ observations of the Tarantula Nebula [98], an age of 3.5 Myr would be inferred from a comparison between the inferred $\mathrm{H} \alpha$ equivalent width of $1100 \AA$ and population synthesis models for a coeval population at LMC metallicity [12]. This is in reasonable agreement with the typical age of massive stars, albeit failing to reflect the complexity in its star-formation history (recall Figure 3).

An analysis of the integrated UV spectrum of NGC 2070 supports a young ( $\leq 3 \mathrm{Myr}$ ) starburst episode [99], while the high spatial resolution of HST/STIS has permitted a comparison between the individual and integrated UV spectroscopic appearance of the central R136 cluster [5]. Very massive stars contributed a significant fraction of its far UV continuum flux, and completely dominate the strong, broad He II $\lambda 1640$ emission. The integrated UV spectroscopic appearance of R136 closely resembles some star clusters in star-forming galaxies at Mpc distances, such as NGC5253-5 [100], suggesting the presence of VMS in these other young massive clusters. From comparison with the predictions of standard population synthesis models, both Starburst99 [101] and BPASS [102] models fail to predict any significant emission prior to the conventional Wolf-Rayet phase (Figure 7), owing to the use of inadequate wind theory for VMS $[93,94]$. Consequently, neither Starburst99 nor BPASS accounts for the powerful winds of very massive stars in R136, and the adopted mass function follows a Salpeter slope, rather than the top heavy IMF identified by [40] for the Tarantula region as a whole.

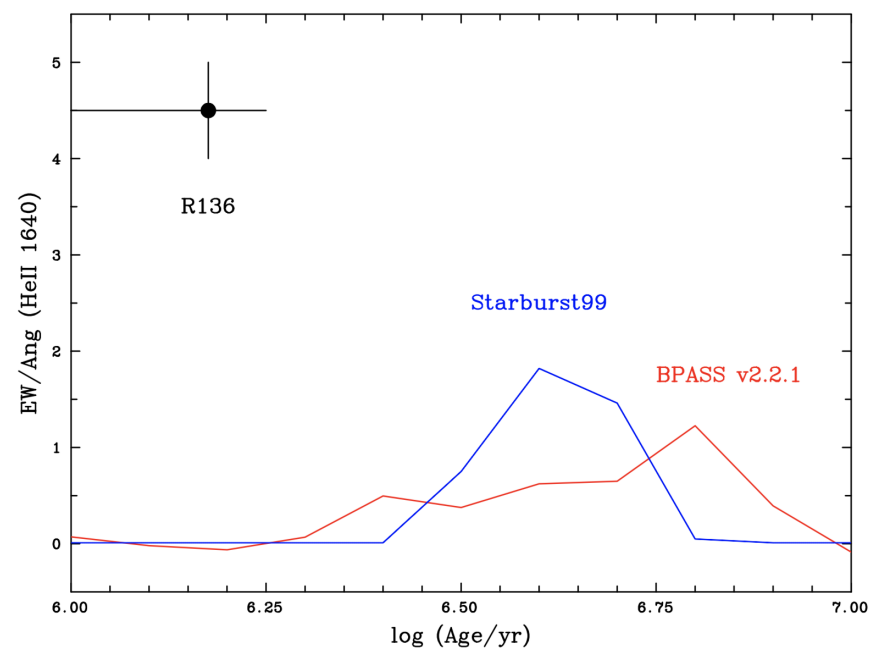

Figure 7. Comparison between observed He II $\lambda 1640$ emission equivalent widths in R136 [5] versus predicted emission from BPASS (v.2.2.1, red) and Starburst99 (blue) population synthesis models (absorption lines are shown as negative values).

An estimate of the cumulative ionizing and mechanical feedback from massive stars within the Tarantula has revealed a major contribution from VMS towards the collective ionizing output and a dominant role from WR stars to the mechanical feedback [12]. However this analysis relied on calibrations and estimates of spectral types for a significant subset of the massive star content, so we are able to provide updates from recent spectroscopic observations (e.g., VLT/MUSE, HST/STIS) and analyses. We present the updated cumulative ionizing output from 1170 massive stars in the Tarantula Nebula in Figure 8, indicating a total Lyman continuum ionizing output of $1.2 \times 10^{52} \mathrm{ph} \mathrm{s}^{-1}$ within 150 pc of R136a. A quarter of the total ionizing radiation originates from the R136a cluster, while 
members of NGC 2070 produce three quarters of the global feedback. Ten systems alone, listed in Table 4, collectively contribute a quarter of the ionizing budget of the Tarantula Nebula, comprising main sequence and classical WR stars, plus early $\mathrm{O}$ supergiants. Indeed, half of the global ionizing output originates from 40 early $\mathrm{O}$ stars, main sequence WN stars and classical WR stars, with 1130 stars contributing the remaining $50 \%$. The collective bolometric luminosity of these stars is $10^{8.4} L_{\odot}$, of which the $40 \mathrm{UV}$-bright stars contribute $10^{8.0} L_{\odot}$.

Table 4. Top ten stellar systems contributing to the Lyman continuum output of the Tarantula Nebula, comprising very massive early O stars and WN5 stars, and classical Wolf-Rayet stars, updated from [12].

\begin{tabular}{lcccl}
\hline Star (Alias) & Sp Type & $\log L / L \odot$ & $\log$ N(LyC) & Reference \\
\hline R136a1 (BAT99-108) & WN5h & 6.8 & 50.6 & {$[55]$} \\
Mk34 (BAT99-116) & WN5h + WN5h & $6.4+6.4$ & 50.6 & {$[37]$} \\
R136a2 (BAT99-109) & WN5h & 6.7 & 50.5 & {$[55]$} \\
R144 (BAT99-118) & WN5-6 + WN6-7 & 6.7 & 50.5 & {$[56,64]$} \\
R1363 (BAT99-106) & WN5h & 6.6 & 50.5 & {$[55]$} \\
Mk49 (BAT99-98) & WN6(h) & 6.7 & 50.5 & {$[56]$} \\
R145 (BAT99-119) & WN6h + O3.5If/WN7 & $6.3+6.3$ & 50.4 & {$[59]$} \\
Mk42 (BAT99-105) & O2 If & 6.6 & 50.4 & {$[51]$} \\
VFTS 682 & WN5h & 6.5 & 50.4 & {$[51]$} \\
R136c (BAT99-112) & WN5h +? & 6.6 & 50.4 & {$[51]$} \\
\hline
\end{tabular}

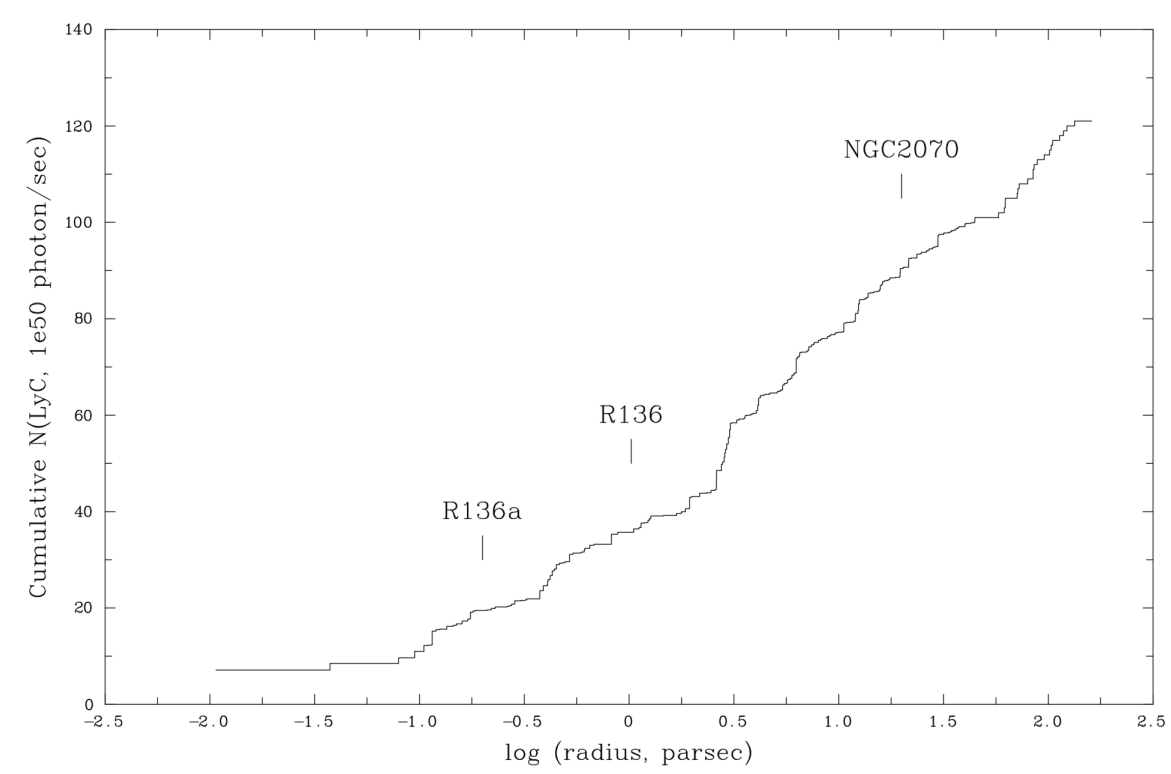

Figure 8. Cumulative ionizing output $\left(10^{50} \mathrm{ph} / \mathrm{s}\right)$ from spectroscopically classified early-type stars in the Tarantula, obtained from VFTS [50,51,53], VLT/MUSE [11], HST/STIS [55] and literature results [56], updated from [12]. Specific regions within 30 Dor are indicated from Table 1.

Recalling Table 2, the highest mass stars and evolved high mass stars in other giant H II regions in the Local Group dominate their radiative and mechanical feedback, including NGC 3372 (Carina Nebula) in the Milky Way, N206 in the LMC and NGC 346 in the SMC. By way of example, Smith [39] established that only a handful of early O-type stars and H-rich WN stars contribute the majority of the Lyman continuum flux of the Carina Nebula, while $\eta$ Car, four Wolf-Rayet stars and two early $\mathrm{O}$ supergiants completely dominate the stellar mechanical luminosity. The central ionizing cluster of the Galactic NGC 3603 star-forming region is host to a stellar content analogous to R136a, including a number of early O stars, nitrogen-sequence Wolf-Rayet stars $[103,104]$. Weak main-sequence wind properties of metal-poor massive stars conspire to even fewer massive stars 
(HD 5980, Sk 80) dominating the cumulative stellar feedback in NGC 346. Similar conclusions were reached by Ramachandran et al. [105] for the supergiant shell in the wing of the SMC.

Although the Tarantula Nebula is the most extreme giant H II region in the Local Group, how does it rank against star-forming regions of galaxies in the near universe or knots at high redshift? Figure 9 compares the star-formation rate versus size of regions spanning $z=0$ to 3.4, adapted from [106], indicating that the Tarantula (red square) is forming stars more vigorously than typical low-redshift counterparts, resembling some star-forming regions at high redshift. Indeed, $80 \%$ of the cumulative ionizing radiation originates from NGC 2070, such that this region corresponds closely with typical clumps in the lensed galaxy SDSS J1110 +6459 at $z=2.5$ (green circles).

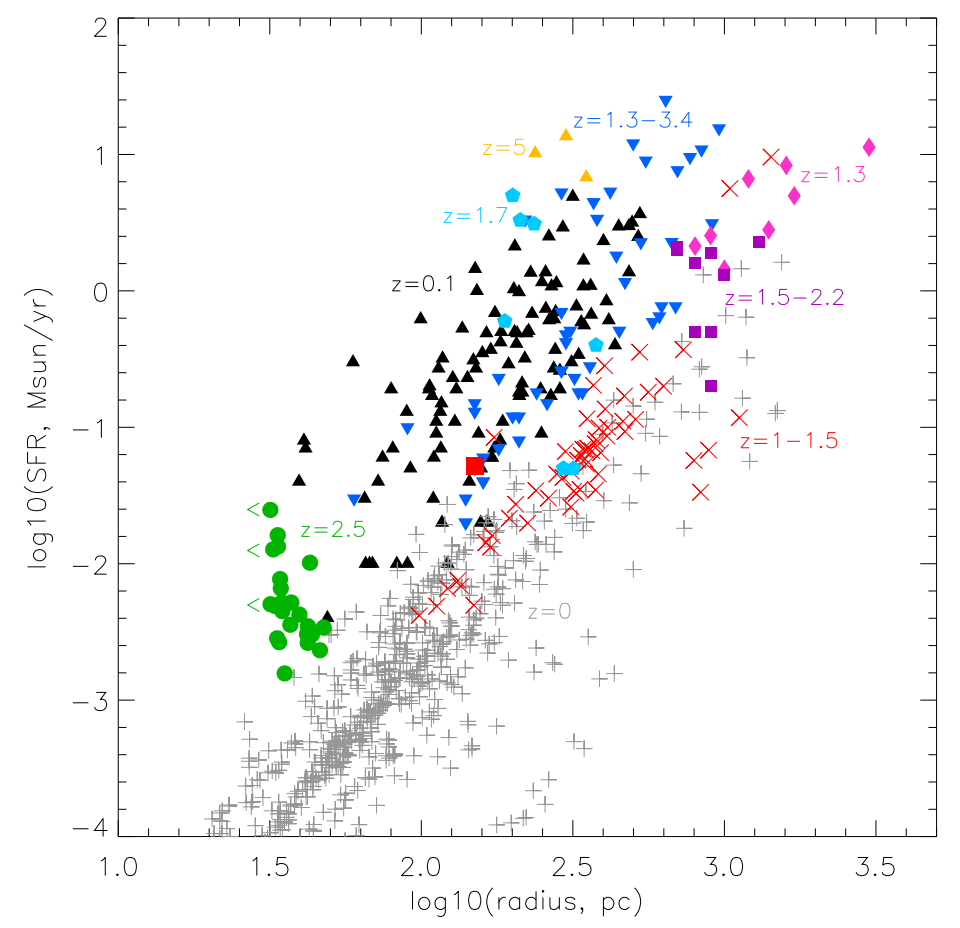

Figure 9. Comparison between the integrated star-formation rate versus size of the Tarantula (filled red square) and star-forming knots from galaxies spanning a range of redshifts, adapted from [106].

In addition to its unusually high star formation rate, the Tarantula also possesses high ionization parameter nebular properties with respect to star-forming galaxies in the local universe. Figure 10 presents a Baldwin, Philipps and Terlevich [107] (BPT) diagnostic diagram of Sloan Digital Sky Survey (SDSS) galaxies, in which the Tarantula (red square) has been indicated, along with Green Pea galaxies from Micheva et al. [108] which are low-metallicity, intensively star-forming galaxies exhibiting unusually strong [O III] $\lambda 5007$ emission. Steidel et al. [109] showed that $z=2-3$ star forming galaxies share similar extreme nebular properties, and a subset of Green Pea galaxies have been established as Lyman continuum leakers [110,111]. Focusing again on NGC 2070, this sits amongst the extreme Green Pea galaxies in the BPT diagram. 


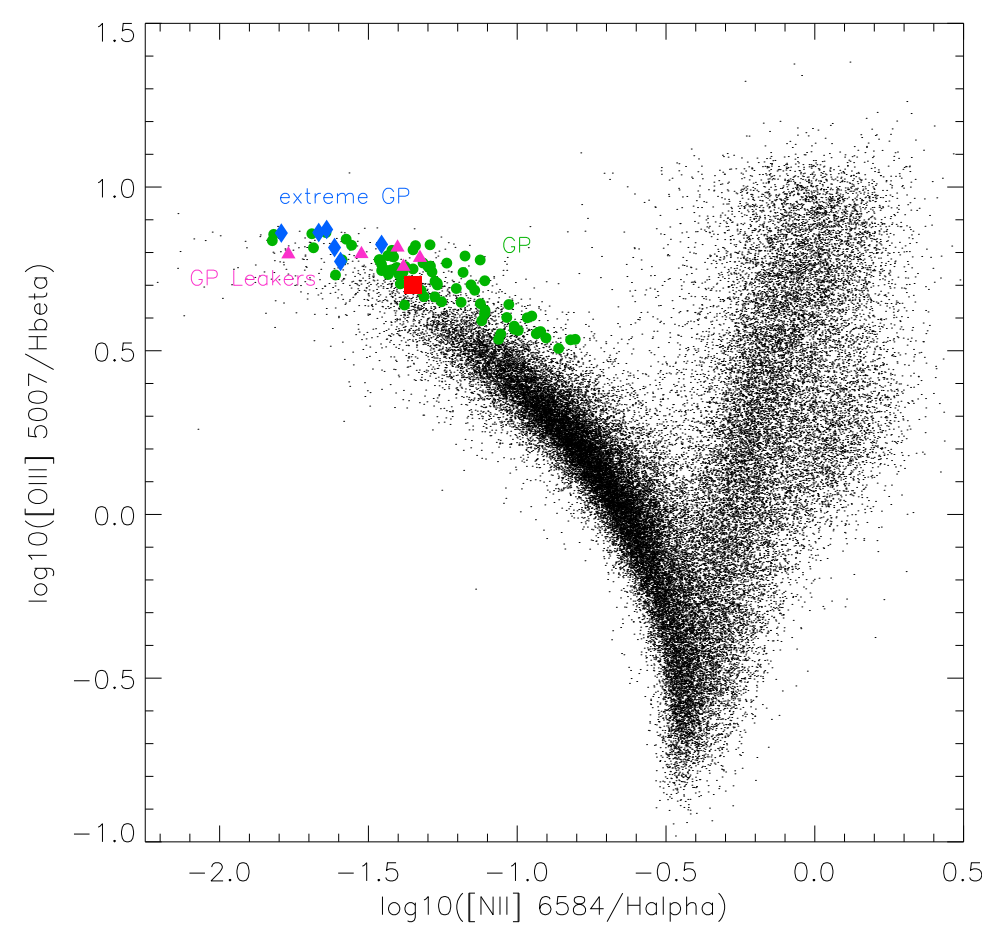

Figure 10. BPT diagram illustrating the similarity in integrated strengths between the Tarantula Nebula (red square), Green Pea (green circles), extreme Green Peas (blue diamonds), Lyman-continuum leaking Green Peak (pink triangles), updated from [108], plus SDSS star-forming galaxies.

\section{Summary and Outlook}

Recent comprehensive spectroscopic and imaging surveys have revealed that the Tarantula Nebula hosts the most exceptional massive star population within the Local Group of galaxies, including the most massive stars identified to date, the fastest rotating early-type stars, and the X-ray brightest colliding wind system. In particular, the VFTS survey has revealed an excess of massive stars with respect to a Salpeter IMF [40] and added support from previous results for the importance of close binary evolution in the evolution of massive stars [64]. As such, the Tarantula Nebula is the closest analogue to the Hubble Deep Field for the community interested in the evolution of massive stars since its richness provides us with a huge breadth of extreme stars.

The integrated appearance of R136 resembles young extragalactic star clusters, while the integrated nebular properties of NGC 2070 is analogous to extreme Green Pea galaxies at low redshift and star-forming knots in high-redshift galaxies. Typical metallicities of Green Pea galaxies are lower than the LMC, as measured from oxygen nebular lines, while the oxygen content of high- $z$ star forming galaxies tends to be similar to those of the Magellanic Clouds. However, $\alpha / \mathrm{Fe}$ abundances of high redshift galaxies are likely to be higher than young populations in the Milky Way, such that winds from OB stars at high redshift are anticipated to be weaker than in the LMC or SMC [112].

The LMC metallicity is only a factor of two below that of the Solar neighbourhood [3], so ideally we would like to supplement the extensive survey of the Tarantula Nebula with counterparts at significantly lower metallicity. The SMC (1/5 solar) represents our best opportunity to study the formation and evolution of massive stars at a metallicity significantly below that of the LMC. Alas, it does not host as rich a massive star-forming region as the Tarantula, but cumulatively does host a substantial number of $\mathrm{O}$ stars so is key towards our improved understanding of massive stars at low metallicity, especially as it can be studied in exquisite detail with current instrumentation. 
Since we need to look beyond the SMC to study metal-poor counterparts to the Tarantula Nebula, Figure 11 compares the star-formation rate, metallicity, and distance modulus of Local Group dwarf galaxies. Rates of star formation in metal-poor ( $\leq 20 \%$ of solar oxygen content) galaxies are significantly lower than the Magellanic Clouds, so there are no rich metal-poor massive star populations elsewhere in the Local Group, and those few that are present are much more distant. The absence of nearby metal-poor counterparts to the Tarantula implies that we currently have to rely on the interpretation of integrated populations in order to understand massive stellar evolution at low metallicity, notably extremely metal-poor dwarf star-forming galaxies I Zw 18 and SBS-0335.

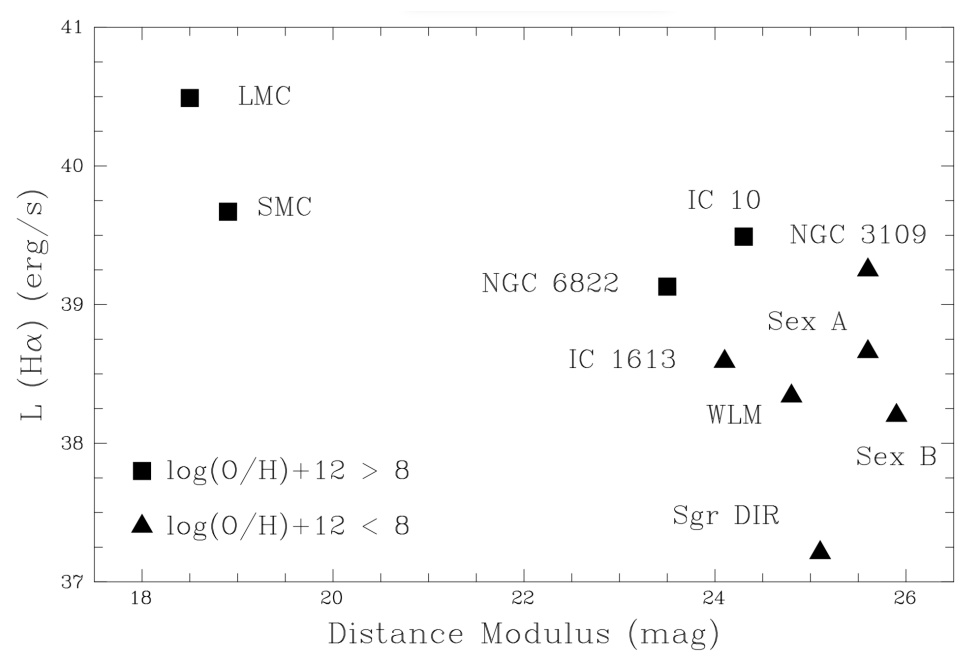

Figure 11. Comparison between present-day star formation rates, as measured by $\mathrm{H} \alpha$ luminosity [113], distance modulus (mag), and oxygen metal content (squares: $\geq 20 \%$ of solar value, triangles: $<20 \%$ of solar value, for Local Group dwarf galaxies. Metal-poor galaxies possess low star-formation rates, so host small numbers of $\mathrm{OB}$ stars, and these are $\geq 6$ magnitudes fainter than Magellanic Cloud counterparts.

In order to test predictions of the metallicity dependence of massive star winds, it is necessary to measure mass-loss properties across a wide range of metallicities. Although theory has been qualitatively supported from the observed wind properties of Milky Way, LMC and SMC early-type stars [82], some issues remain, including weak winds in low luminosity OB stars. Our only opportunity to study individual massive stars below $1 / 10$ th of the solar oxygen content is to observe $\mathrm{O}$ stars in Sextans A and B with 7\% solar [114] or the Sagittarius Dwarf Irregular Galaxy (SgrDIR) with 5\% solar [115]. Stellar winds from early-type stars at such low metallicities are anticipated to be much weaker than in metal-rich populations, which has been confirmed by UV spectroscopy. By way of example, Figure 12 compares the far UV spectrum of $\xi$ Per (O7.5 III) with a counterpart in Sextans A [116], revealing negligible wind signatures in the latter (e.g., C IV $\lambda 1550)$. 


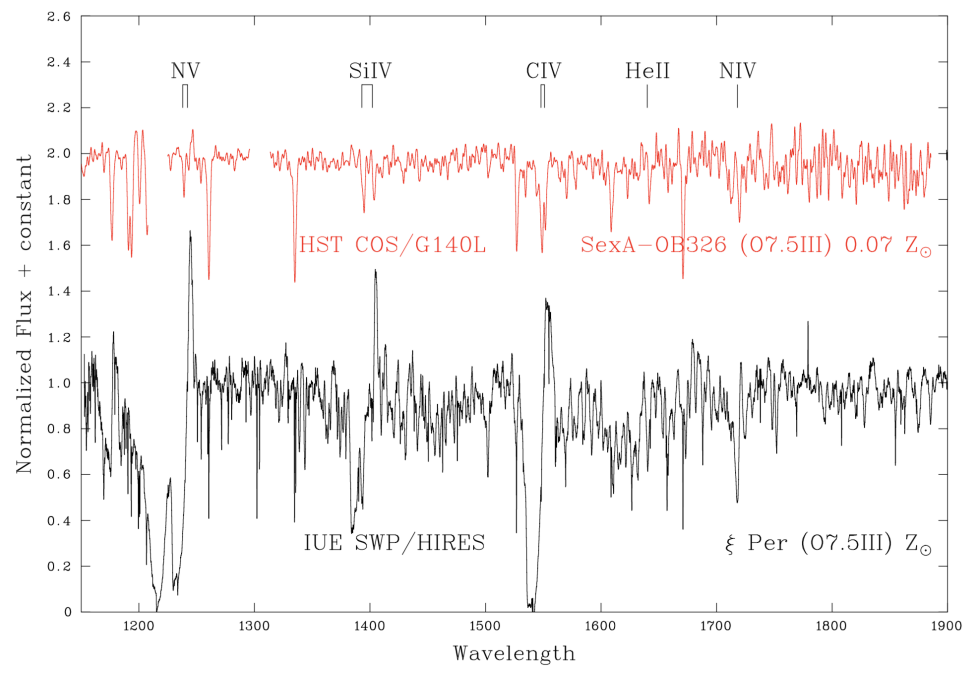

Figure 12. Comparison between far UV spectroscopy of mid O giants in metal-rich [117] and metal-deficient [116] environments, illustrating the extreme differences in wind features (e.g., $\mathrm{N} ; \mathrm{V}$ $\lambda 1240$, Si IV $\lambda 1400$, C IV $\lambda 1550)$ and the iron forest (Fe IV-V).

Funding: This research received no external funding.

Acknowledgments: This review is dedicated to the memory of Nolan Walborn, from whom the author learnt a great deal about the Tarantula Nebula. Thanks to Leisa Townsley and Patrick Broos for access to T-ReX point source results prior to publication, Miriam Garcia for the UV spectrum of Sextans A OB 326, Selma De Mink for the VFTS pie chart, Heloise Stevance for the BPASS predictions, and Joachim Bestenlehner for converting $Q$ wind density results into mass-loss rates. Feedback from Fabian Schneider, Chris Evans, Joachim Bestenlehner, Andy Pollock, Roberta Humphreys and external referees on an earlier draft is greatly appreciated.

Conflicts of Interest: The author declares no conflict of interest.

\section{References}

1. Kennicutt, R.C., Jr. Star Formation in Galaxies Along the Hubble Sequence. Ann. Rev. Astron. Astrophys. 1998, 36, 189-232. [CrossRef]

2. Evans, C.J.; Taylor, W.D.; Hénault-Brunet, V.; Sana, H.; de Koter, A.; Simón-Díaz, S.; Carraro, G.; Bagnoli, T.; Bastian, N.; Bestenlehner, J.M.; et al. The VLT-FLAMES Tarantula Survey. I. Introduction and observational overview. Astron. Astrophys. 2011, 530, A108. [CrossRef]

3. Tsamis, Y.G.; Péquignot, D. A photoionization-modelling study of 30 Doradus: The case for small-scale chemical inhomogeneity. Mon. Not. R. Astron. Soc. 2005, 364, 687-704. [CrossRef]

4. Baldwin, J.A.; Ferland, G.J.; Martin, P.G.; Corbin, M.R.; Cota, S.A.; Peterson, B.M.; Slettebak, A. Physical conditions in the Orion Nebula and an assessment of its helium abundance. Astrophys. J. 1991, 374, 580-609. [CrossRef]

5. Crowther, P.A.; Caballero-Nieves, S.M.; Bostroem, K.A.; Maíz Apellániz, J.; Schneider, F.R.N.; Walborn, N.R.; Angus, C.R.; Brott, I.; Bonanos, A.; de Koter, A.; et al. The R136 star cluster dissected with Hubble Space Telescope/STIS. I. Far-ultraviolet spectroscopic census and the origin of He II $\lambda 1640$ in young star clusters. Mon. Not. R. Astron. Soc. 2016, 458, 624-659. [CrossRef]

6. Britavskiy, N.; Lennon, D.J.; Patrick, L.R.; Evans, C.J.; Herrero, A.; Langer, N.; van Loon, J.T.; Clark, J.S.; Schneider, F.R.N.; Almeida, L.A.; et al. The VLT-FLAMES Tarantula Survey. XXX. Red stragglers in the clusters Hodge 301 and SL 639. Astron. Astrophys. 2019, 624, A128. [CrossRef]

7. Walborn, N.R. The Starburst Region 30 Doradus. In The Magellanic Clouds: Proceedings of the 148th Symposium of the International Astronomical UnionThe Magellanic Clouds; Haynes, R., Milne, D., Eds.; Kluwer Academic Publishers: Dordrecht, The Netherland, 1991; Volume 148, p. 145.

8. Sabbi, E.; Anderson, J.; Lennon, D.J.; van der Marel, R.P.; Aloisi, A.; Boyer, M.L.; Cignoni, M.; de Marchi, G.; de Mink, S.E.; Evans, C.J.; et al. Hubble Tarantula Treasury Project: Unraveling Tarantula's Web. I. Observational Overview and First Results. Astron. J. 2013, 146, 53. [CrossRef] 
9. Crowther, P.A.; Schnurr, O.; Hirschi, R.; Yusof, N.; Parker, R.J.; Goodwin, S.P.; Kassim, H.A. The R136 star cluster hosts several stars whose individual masses greatly exceed the accepted $150 \mathrm{M}_{\text {solar }}$ stellar mass limit. Mon. Not. R. Astron. Soc. 2010, 408, 731-751. [CrossRef]

10. Castro, N.; Crowther, P.A.; Evans, C.J.; Mackey, J.; Castro-Rodriguez, N.; Vink, J.S.; Melnick, J.; Selman, F. Mapping the core of the Tarantula Nebula with VLT-MUSE. I. Spectral and nebular content around R136. Astron. Astrophys. 2018, 614, A147. [CrossRef]

11. Castro, N.; Crowther, P.A.; Evans, C.J.; Mackey, J.; Castro-Rodriguez, N.; Vink, J.S.; Melnick, J.; Selman, F. Mapping the core of the Tarantula Nebula with VLT-MUSE. III. The spectroscopic Hertzsprung-Russell diagram of OB stars in NGC2070. Astron. Astrophys. in preparation.

12. Doran, E.I.; Crowther, P.A.; de Koter, A.; Evans, C.J.; McEvoy, C.; Walborn, N.R.; Bastian, N.; Bestenlehner, J.M.; Gräfener, G.; Herrero, A.; et al. The VLT-FLAMES Tarantula Survey. XI. A census of the hot luminous stars and their feedback in 30 Doradus. Astron. Astrophys. 2013, 558, A134. [CrossRef]

13. De Marchi, G.; Paresce, F.; Panagia, N.; Beccari, G.; Spezzi, L.; Sirianni, M.; Andersen, M.; Mutchler, M.; Balick, B.; Dopita, M.A.; et al. Star Formation in 30 Doradus. Astrophys. J. 2011, 739, 27. [CrossRef]

14. Sabbi, E.; Lennon, D.J.; Anderson, J.; Cignoni, M.; van der Marel, R.P.; Zaritsky, D.; De Marchi, G.; Panagia, N.; Gouliermis, D.A.; Grebel, E.K.; et al. Hubble Tarantula Treasury Project. III. Photometric Catalog and Resulting Constraints on the Progression of Star Formation in the 30 Doradus Region. Astrophys. J. 2016, 222, 11. [CrossRef]

15. Schneider, F.R.N.; Ramírez-Agudelo, O.H.; Tramper, F.; Bestenlehner, J.M.; Castro, N.; Sana, H.; Evans, C.J.; Sabín-Sanjulián, C.; Simón-Díaz, S.; Langer, N.; et al. The VLT-FLAMES Tarantula Survey. XXIX. Massive star formation in the local 30 Doradus starburst. Astron. Astrophys. 2018, 618, A73. [CrossRef]

16. Wright, N.J.; Bouy, H.; Drew, J.E.; Sarro, L.M.; Bertin, E.; Cuillandre, J.C.; Barrado, D. Cygnus OB2 DANCe: A high-precision proper motion study of the Cygnus OB2 association. Mon. Not. R. Astron. Soc. 2016, 460, 2593-2610. [CrossRef]

17. Walborn, N.R. The Pillars of the Second Generation. In Hot Star Workshop III: The Earliest Phases of Massive Star Birth; Astronomical Society of the Pacific Conference Series; Crowther, P.A., Ed.; Astronomical Society of the Pacific: San Francisco, CA, USA, 2002; Volume 267, p. 111.

18. Walborn, N.R.; Barbá, R.H.; Sewiło, M.M. The Top 10 Spitzer Young Stellar Objects in 30 Doradus. Astron. J. 2013, 145, 98. [CrossRef]

19. Indebetouw, R.; Brogan, C.; Chen, C.H.R.; Leroy, A.; Johnson, K.; Muller, E.; Madden, S.; Cormier, D.; Galliano, F.; Hughes, A.; et al. ALMA Resolves 30 Doradus: Sub-parsec Molecular Cloud Structure near the Closest Super Star Cluster. Astrophys. J. 2013, 774, 73. [CrossRef]

20. Kennicutt, R.C., Jr. Structural properties of giant H II regions in nearby galaxies. Astrophys. J. 1984, 287, 116-130. [CrossRef]

21. Parker, J.W. The OB associations of 30 Doradus in the Large Magellanic Cloud. I-Stellar observations and data reductions. Astron. J. 1993, 106, 560-577. [CrossRef]

22. Parker, J.W.; Garmany, C.D. The OB associations of 30 Doradus in the Large Magellanic Cloud. II-Stellar content and initial mass function. Astron. J. 1993, 106, 1471-1483. [CrossRef]

23. Massey, P.; Hunter, D.A. Star Formation in R136: A Cluster of O3 Stars Revealed by Hubble Space Telescope Spectroscopy. Astrophys. J. 1998, 493, 180-194. [CrossRef]

24. Bosch, G.; Terlevich, R.; Melnick, J.; Selman, F. The ionising cluster of 30 Doradus. II. Spectral classification for 175 stars. Astron. Astrophys. 1999, 137, 21-41. [CrossRef]

25. Selman, F.; Melnick, J.; Bosch, G.; Terlevich, R. The ionizing cluster of 30 Doradus. III. Star-formation history and initial mass function. Astron. Astrophys. 1999, 347, 532-549.

26. Breysacher, J.; Azzopardi, M.; Testor, G. The fourth catalogue of Population I Wolf-Rayet stars in the Large Magellanic Cloud Astron. Astrophys. 1999 137, 117-145.

27. Walborn, N.R.; Gamen, R.C.; Morrell, N.I.; Barbá, R.H.; Fernández Lajús, E.; Angeloni, R. Active Luminous Blue Variables in the Large Magellanic Cloud. Astron. J. 2017, 154, 15. [CrossRef]

28. Lennon, D.J.; Evans, C.J.; van der Marel, R.P.; Anderson, J.; Platais, I.; Herrero, A.; de Mink, S.E.; Sana, H.; Sabbi, E.; Bedin, L.R.; et al. Gaia DR2 reveals a very massive runaway star ejected from R136. Astron. Astrophys. 2018, 619, A78. [CrossRef] 
29. Dufton, P.L.; Dunstall, P.R.; Evans, C.J.; Brott, I.; Cantiello, M.; de Koter, A.; de Mink, S.E.; Fraser, M.; Hénault-Brunet, V.; Howarth, I.D.; et al. The VLT-FLAMES Tarantula Survey: The Fastest Rotating O-type Star and Shortest Period LMC Pulsar: Remnants of a Supernova Disrupted Binary? Astrophys. J. Lett. 2011, 743, L22. [CrossRef]

30. Almeida, L.A.; Sana, H.; de Mink, S.E.; Tramper, F.; Soszyński, I.; Langer, N.; Barbá, R.H.; Cantiello, M.; Damineli, A.; de Koter, A.; et al. Discovery of the Massive Overcontact Binary VFTS352: Evidence for Enhanced Internal Mixing. Astrophys. J. 2015, 812, 102. [CrossRef]

31. Chen, Y.; Wang, Q.D.; Gotthelf, E.V.; Jiang, B.; Chu, Y.H.; Gruendl, R. Chandra ACIS Spectroscopy of N157B: A Young Composite Supernova Remnant in a Superbubble. Astrophys. J. 2006, 651, 237-249. [CrossRef]

32. Savage, B.D.; Fitzpatrick, E.L.; Cassinelli, J.P.; Ebbets, D.C. The nature of R136a, the superluminous central object of the 30 Doradus nebula. Astrophys. J. 1983, 273, 597-623. [CrossRef]

33. Weigelt, G.; Baier, G. R136a in the 30 Doradus nebula resolved by holographic speckle interferometry. Astron. Astrophys. 1985, 150, L18-L20.

34. Hunter, D.A.; Shaya, E.J.; Holtzman, J.A.; Light, R.M.; O’Neil, E.J., Jr.; Lynds, R. The Intermediate Stellar Mass Population in R136 Determined from Hubble Space Telescope Planetary Camera 2 Images. Astrophys. J. 1995, 448, 179. [CrossRef]

35. de Koter, A.; Heap, S.R.; Hubeny, I. On the Evolutionary Phase and Mass Loss of the Wolf-Rayet-like Stars in R136a. Astrophys. J. 1997, 477, 792. [CrossRef]

36. Khorrami, Z.; Vakili, F.; Lanz, T.; Langlois, M.; Lagadec, E.; Meyer, M.R.; Robbe-Dubois, S.; Abe, L.; Avenhaus, H.; Beuzit, J.L.; et al. Uncrowding R 136 from VLT/SPHERE extreme adaptive optics. Astron. Astrophys. 2017, 602, A56. [CrossRef]

37. Tehrani, K.A.; Crowther, P.A.; Bestenlehner, J.M.; Littlefair, S.P.; Pollock, A.M.T.; Parker, R.J.; Schnurr, O. Weighing Melnick 34: The most massive binary system known. Mon. Not. R. Astron. Soc. 2019, 484, 2692-2710. [CrossRef]

38. Pollock, A.M.T.; Crowther, P.A.; Tehrani, K.; Broos, P.S.; Townsley, L.K. The 155-day X-ray cycle of the very massive Wolf-Rayet star Melnick 34 in the Large Magellanic Cloud. Mon. Not. R. Astron. Soc. 2018, 474, 3228-3236. [CrossRef]

39. Smith, N. A census of the Carina Nebula - I. Cumulative energy input from massive stars. Mon. Not. R. Astron. Soc. 2006, 367, 763-772. [CrossRef]

40. Schneider, F.R.N.; Sana, H.; Evans, C.J.; Bestenlehner, J.M.; Castro, N.; Fossati, L.; Gräfener, G.; Langer, N.; Ramírez-Agudelo, O.H.; Sabín-Sanjulián, C.; et al. An excess of massive stars in the local 30 Doradus starburst. Science 2018, 359, 69-71. [CrossRef]

41. Puls, J.; Urbaneja, M.A.; Venero, R.; Repolust, T.; Springmann, U.; Jokuthy, A.; Mokiem, M.R. Atmospheric NLTE-models for the spectroscopic analysis of blue stars with winds. II. Line-blanketed models. Astron. Astrophys. 2005, 435, 669-698. [CrossRef]

42. Hillier, D.J.; Miller, D.L. The Treatment of Non-LTE Line Blanketing in Spherically Expanding Outflows. Astrophys. J. 1998, 496, 407-427. [CrossRef]

43. Gräfener, G.; Koesterke, L.; Hamann, W.R. Line-blanketed model atmospheres for WR stars. Astron. Astrophys. 2002, 387, 244-257. [CrossRef]

44. Lanz, T.; Hubeny, I. A Grid of NLTE Line-blanketed Model Atmospheres of Early B-Type Stars. Astrophys. J. Suppl. 2007, 169, 83-104. [CrossRef]

45. Gustafsson, B.; Edvardsson, B.; Eriksson, K.; Jørgensen, U.G.; Nordlund, Å.; Plez, B. A grid of MARCS model atmospheres for late-type stars. I. Methods and general properties. Astron. Astrophys. 2008, 486, 951-970. [CrossRef]

46. Langer, N.; Kudritzki, R.P. The spectroscopic Hertzsprung-Russell diagram. Astron. Astrophys. 2014, 564, A52. [CrossRef]

47. Gordon, M.S.; Humphreys, R.M.; Jones, T.J. Luminous and Variable Stars in M31 and M33. III. The Yellow and Red Supergiants and Post-red Supergiant Evolution. Astrophys. J. 2016, 825, 50. [CrossRef]

48. Davies, B.; Crowther, P.A.; Beasor, E.R. The luminosities of cool supergiants in the Magellanic Clouds, and the Humphreys-Davidson limit revisited. Mon. Not. R. Astron. Soc. 2018, 478, 3138-3148. [CrossRef]

49. Schneider, F.R.N.; Langer, N.; de Koter, A.; Brott, I.; Izzard, R.G.; Lau, H.H.B. Bonnsai: A Bayesian tool for comparing stars with stellar evolution models. Astron. Astrophys. 2014, 570, A66. [CrossRef] 
50. Sabín-Sanjulián, C.; Simón-Díaz, S.; Herrero, A.; Puls, J.; Schneider, F.R.N.; Evans, C.J.; Garcia, M.; Najarro, F.; Brott, I.; Castro, N.; et al. The VLT-FLAMES Tarantula Survey. XXVI. Properties of the O-dwarf population in 30 Doradus. Astron. Astrophys. 2017, 601, A79. [CrossRef]

51. Bestenlehner, J.M.; Gräfener, G.; Vink, J.S.; Najarro, F.; de Koter, A.; Sana, H.; Evans, C.J.; Crowther, P.A.; Hénault-Brunet, V.; Herrero, A.; et al. The VLT-FLAMES Tarantula Survey. XVII. Physical and wind properties of massive stars at the top of the main sequence. Astron. Astrophys. 2014, 570, A38. [CrossRef]

52. McEvoy, C.M.; Dufton, P.L.; Evans, C.J.; Kalari, V.M.; Markova, N.; Simón-Díaz, S.; Vink, J.S.; Walborn, N.R.; Crowther, P.A.; de Koter, A.; et al. The VLT-FLAMES Tarantula Survey. XIX. B-type supergiants: Atmospheric parameters and nitrogen abundances to investigate the role of binarity and the width of the main sequence. Astron. Astrophys. 2015, 575, A70. [CrossRef]

53. Ramírez-Agudelo, O.H.; Sana, H.; de Koter, A.; Tramper, F.; Grin, N.J.; Schneider, F.R.N.; Langer, N.; Puls, J.; Markova, N.; Bestenlehner, J.M.; et al. The VLT-FLAMES Tarantula Survey. XXIV. Stellar properties of the O-type giants and supergiants in 30 Doradus. Astron. Astrophys. 2017, 600, A81. [CrossRef]

54. Dufton, P.L.; Thompson, A.; Crowther, P.A.; Evans, C.J.; Schneider, F.R.N.; de Koter, A.; de Mink, S.E.; Garland, R.; Langer, N.; Lennon, D.J.; et al. The VLT-FLAMES Tarantula Survey. XXVIII. Nitrogen abundances for apparently single dwarf and giant B-type stars with small projected rotational velocities. Astron. Astrophys. 2018, 615, A101. [CrossRef]

55. Bestenlehner, J.M.; Crowther, P.; Caballero-Nieves, S.M.; Simón-Díaz, S.; Schneider, F. The R136 star cluster dissected with Hubble Space Telescope.III Physical properties of the most massive stars in R136. Mon. Not. R. Astron. Soc. in preparation.

56. Hainich, R.; Rühling, U.; Todt, H.; Oskinova, L.M.; Liermann, A.; Gräfener, G.; Foellmi, C.; Schnurr, O.; Hamann, W.R. The Wolf-Rayet stars in the Large Magellanic Cloud. A comprehensive analysis of the WN class. Astron. Astrophys. 2014, 565, A27. [CrossRef]

57. Garland, R.; Dufton, P.L.; Evans, C.J.; Crowther, P.A.; Howarth, I.D.; de Koter, A.; de Mink, S.E.; Grin, N.J.; Langer, N.; Lennon, D.J.; et al. The VLT-FLAMES Tarantula Survey. XXVII. Physical parameters of B-type main-sequence binary systems in the Tarantula nebula. Astron. Astrophys. 2017, 603, A91. [CrossRef]

58. Mahy, L.; Sana, H.; Abdul-Masih, M.; Almeida, L.A.; Langer, N.; Shenar, T.; de Koter, A.; de Mink, S.; de Wit, S.; Grin, N.; et al. The Tarantula Massive Binary Monitoring: III. Atmosphere analysis of double-lined spectroscopic systems. Astron. Astrophys. submitted.

59. Shenar, T.; Sablowski, D.P.; Hainich, R.; Todt, H.; Moffat, A.F.J.; Oskinova, L.M.; Ramachandran, V.; Sana, H.; Sander, A.A.C.; Schnurr, O.; et al. The Wolf-Rayet binaries of the nitrogen sequence in the Large Magellanic Cloud. Spectroscopy, orbital analysis, formation, and evolution. Astron. Astrophys. 2019, 627, A151. [CrossRef]

60. Brott, I.; de Mink, S.E.; Cantiello, M.; Langer, N.; de Koter, A.; Evans, C.J.; Hunter, I.; Trundle, C.; Vink, J.S. Rotating massive main-sequence stars. I. Grids of evolutionary models and isochrones. Astron. Astrophys. 2011, 530, A115. [CrossRef]

61. Köhler, K.; Langer, N.; de Koter, A.; de Mink, S.E.; Crowther, P.A.; Evans, C.J.; Gräfener, G.; Sana, H.; Sanyal, D.; Schneider, F.R.N.; et al. The evolution of rotating very massive stars with LMC composition. Astron. Astrophys. 2015, 573, A71. [CrossRef]

62. Vanbeveren, D.; De Loore, C.; Van Rensbergen, W. Massive stars. Astron. Astrophys. Rev. 1998, 9, 63-152. [CrossRef]

63. Sana, H.; de Mink, S.E.; de Koter, A.; Langer, N.; Evans, C.J.; Gieles, M.; Gosset, E.; Izzard, R.G.; Le Bouquin, J.B.; Schneider, F.R.N. Binary Interaction Dominates the Evolution of Massive Stars. Science 2012, 337, 444. [CrossRef] [PubMed]

64. Sana, H.; de Koter, A.; de Mink, S.E.; Dunstall, P.R.; Evans, C.J.; Hénault-Brunet, V.; Maíz Apellániz, J.; Ramírez-Agudelo, O.H.; Taylor, W.D.; Walborn, N.R.; et al. The VLT-FLAMES Tarantula Survey. VIII. Multiplicity properties of the O-type star population. Astron. Astrophys. 2013, 550, A107. [CrossRef]

65. Dunstall, P.R.; Dufton, P.L.; Sana, H.; Evans, C.J.; Howarth, I.D.; Simón-Díaz, S.; de Mink, S.E.; Langer, N.; Maíz Apellániz, J.; Taylor, W.D. The VLT-FLAMES Tarantula Survey. XXII. Multiplicity properties of the B-type stars. Astron. Astrophys. 2015, 580, A93. [CrossRef]

66. Almeida, L.A.; Sana, H.; Taylor, W.; Barbá, R.; Bonanos, A.Z.; Crowther, P.; Damineli, A.; de Koter, A.; de Mink, S.E.; Evans, C.J.; et al. The Tarantula Massive Binary Monitoring. I. Observational campaign and OB-type spectroscopic binaries. Astron. Astrophys. 2017, 598, A84. [CrossRef] 
67. Götberg, Y.; de Mink, S.E.; Groh, J.H.; Kupfer, T.; Crowther, P.A.; Zapartas, E.; Renzo, M. Spectral models for binary products: Unifying subdwarfs and Wolf-Rayet stars as a sequence of stripped-envelope stars. Astron. Astrophys. 2018, 615, A78. [CrossRef]

68. Simón-Díaz, S.; Herrero, A. The IACOB project. I. Rotational velocities in northern Galactic O- and early B-type stars revisited. The impact of other sources of line-broadening. Astron. Astrophys. 2014, 562, A135. [CrossRef]

69. Ramírez-Agudelo, O.H.; Simón-Díaz, S.; Sana, H.; de Koter, A.; Sabín-Sanjulían, C.; de Mink, S.E.; Dufton, P.L.; Gräfener, G.; Evans, C.J.; Herrero, A.; et al. The VLT-FLAMES Tarantula Survey. XII. Rotational velocities of the single O-type stars. Astron. Astrophys. 2013, 560, A29. [CrossRef]

70. Ramírez-Agudelo, O.H.; Sana, H.; de Mink, S.E.; Hénault-Brunet, V.; de Koter, A.; Langer, N.; Tramper, F.; Gräfener, G.; Evans, C.J.; Vink, J.S.; et al. The VLT-FLAMES Tarantula Survey. XXI. Stellar spin rates of O-type spectroscopic binaries. Astron. Astrophys. 2015, 580, A92. [CrossRef]

71. Dufton, P.L.; Langer, N.; Dunstall, P.R.; Evans, C.J.; Brott, I.; de Mink, S.E.; Howarth, I.D.; Kennedy, M.; McEvoy, C.; Potter, A.T.; et al. The VLT-FLAMES Tarantula Survey. X. Evidence for a bimodal distribution of rotational velocities for the single early B-type stars. Astron. Astrophys. 2013, 550, A109. [CrossRef]

72. Wolff, S.C.; Strom, S.E.; Cunha, K.; Daflon, S.; Olsen, K.; Dror, D. Rotational Velocities for Early-Type Stars in the Young Large Magellanic Cloud Cluster R136: Further Study of the Relationship Between Rotation Speed and Density in Star-Forming Regions. Astron. J. 2008, 136, 1049-1060. [CrossRef]

73. Platais, I.; Lennon, D.J.; van der Marel, R.P.; Bellini, A.; Sabbi, E.; Watkins, L.L.; Sohn, S.T.; Walborn, N.R.; Bedin, L.R.; Evans, C.J.; et al. HST Astrometry in the 30 Doradus Region. II. Runaway Stars from New Proper Motions in the Large Magellanic Cloud. Astron. J. 2018, 156, 98. [CrossRef]

74. Evans, C.J.; Walborn, N.R.; Crowther, P.A.; Hénault-Brunet, V.; Massa, D.; Taylor, W.D.; Howarth, I.D.; Sana, H.; Lennon, D.J.; van Loon, J.T. A Massive Runaway Star from 30 Doradus. Astrophys. J. Lett. 2010, 715, L74-L79. [CrossRef]

75. Renzo, M.; de Mink, S.E.; Lennon, D.J.; Platais, I.; van der Marel, R.P.; Laplace, E.; Bestenlehner, J.M.; Evans, C.J.; Hénault-Brunet, V.; Justham, S.; et al. Space astrometry of the very massive $150 \mathrm{M}_{\odot}$ candidate runaway star VFTS682. Mon. Not. R. Astron. Soc. 2019, 482, L102-L106. [CrossRef]

76. Pallavicini, R.; Golub, L.; Rosner, R.; Vaiana, G.S.; Ayres, T.; Linsky, J.L. Relations among stellar X-ray emission observed from Einstein, stellar rotation and bolometric luminosity. Astrophys. J. 1981, 248, 279-290. [CrossRef]

77. Stevens, I.R.; Blondin, J.M.; Pollock, A.M.T. Colliding winds from early-type stars in binary systems. Astrophys. J. 1992, 386, 265-287. [CrossRef]

78. Massey, P.; Penny, L.R.; Vukovich, J. Orbits of Four Very Massive Binaries in the R136 Cluster. Astrophys. J. 2002, 565, 982-993. [CrossRef]

79. Taylor, W.D.; Evans, C.J.; Sana, H.; Walborn, N.R.; de Mink, S.E.; Stroud, V.E.; Alvarez-Candal, A.; Barbá, R.H.; Bestenlehner, J.M.; Bonanos, A.Z.; et al. The VLT-FLAMES Tarantula Survey. II. R139 revealed as a massive binary system. Astron. Astrophys. 2011, 530, L10. [CrossRef]

80. Clark, J.S.; Bartlett, E.S.; Broos, P.S.; Townsley, L.K.; Taylor, W.D.; Walborn, N.R.; Bird, A.J.; Sana, H.; de Mink, S.E.; Dufton, P.L.; et al. The VLT-FLAMES Tarantula survey. XX. The nature of the X-ray bright emission-line star VFTS 399. Astron. Astrophys. 2015, 579, A131. [CrossRef]

81. Castor, J.I.; Abbott, D.C.; Klein, R.I. Radiation-driven winds in Of stars. Astrophys. J. 1975, 195, $157-174$. [CrossRef]

82. Mokiem, M.R.; de Koter, A.; Vink, J.S.; Puls, J.; Evans, C.J.; Smartt, S.J.; Crowther, P.A.; Herrero, A.; Langer, N.; Lennon, D.J.; et al. The empirical metallicity dependence of the mass-loss rate of O- and early B-type stars. Astron. Astrophys. 2007, 473, 603-614. [CrossRef]

83. Vink, J.S.; de Koter, A.; Lamers, H.J.G.L.M. Mass-loss predictions for O and B stars as a function of metallicity. Astron. Astrophys. 2001, 369, 574-588. [CrossRef]

84. Prinja, R.K.; Barlow, M.J.; Howarth, I.D. Terminal velocities for a large sample of O stars, B supergiants, and Wolf-Rayet stars. Astrophys. J. 1990, 361, 607-620. [CrossRef]

85. Roman-Duval, J.; Jenkins, E.B.; Williams, B.; Tchernyshyov, K.; Gordon, K.; Meixner, M.; Hagen, L.; Peek, J.; Sandstrom, K.; Werk, J.; et al. METAL: The Metal Evolution, Transport, and Abundance in the Large Magellanic Cloud Hubble Program. I. Overview and Initial Results. Astrophys. J. 2019, 871, 151. [CrossRef] 
86. Schmutz, W.; Hamann, W.R.; Wessolowski, U. Spectral analysis of 30 Wolf-Rayet stars. Astron. Astrophys. 1989, 210, 236-248.

87. St.-Louis, N.; Moffat, A.F.J.; Drissen, L.; Bastien, P.; Robert, C. Polarization variability among Wolf-Rayet stars. III - A new way to derive mass-loss rates for Wolf-Rayet stars in binary systems. Astrophys. J. 1988, 330, 286-304. [CrossRef]

88. Hillier, D.J. The effects of electron scattering and wind clumping for early emission line stars. Astron. Astrophys. 1991, 247, 455-468.

89. Fullerton, A.W.; Massa, D.L.; Prinja, R.K. The Discordance of Mass-Loss Estimates for Galactic O-Type Stars. Astrophys. J. 2006, 637, 1025-1039. [CrossRef]

90. Sundqvist, J.O.; Puls, J. Atmospheric NLTE models for the spectroscopic analysis of blue stars with winds. IV. Porosity in physical and velocity space. Astron. Astrophys. 2018, 619, A59. [CrossRef]

91. Lamers, H.J.G.L.M.; Snow, T.P.; Lindholm, D.M. Terminal Velocities and the Bistability of Stellar Winds. Astrophys. J. 1995, 455, 269. [CrossRef]

92. Kudritzki, R.P.; Puls, J. Winds from Hot Stars. Ann. Rev. Astron. Astrophys. 2000, 38, 613-666. [CrossRef]

93. Gräfener, G.; Vink, J.S.; de Koter, A.; Langer, N. The Eddington factor as the key to understand the winds of the most massive stars. Evidence for a $\Gamma$-dependence of Wolf-Rayet type mass loss. Astron. Astrophys. 2011, 535, A56. [CrossRef]

94. Bestenlehner, J.M. Mass loss and the Eddington factor: An updated stellar wind theory for hot massive stars. Mon. Not. R. Astron. Soc. in preparation.

95. Puls, J.; Vink, J.S.; Najarro, F. Mass loss from hot massive stars. Astron. Astrophys. Rev. 2008, 16, $209-325$. [CrossRef]

96. Langer, N. Presupernova Evolution of Massive Single and Binary Stars. Ann. Rev. Astron. Astrophys. 2012, 50, 107-164. [CrossRef]

97. Yusof, N.; Hirschi, R.; Meynet, G.; Crowther, P.A.; Ekström, S.; Frischknecht, U.; Georgy, C.; Abu Kassim, H.; Schnurr, O. Evolution and fate of very massive stars. Mon. Not. R. Astron. Soc. 2013, 433, 1114-1132. [CrossRef]

98. Pellegrini, E.W.; Baldwin, J.A.; Ferland, G.J. Structure and Feedback in 30 Doradus. I. Observations. Astrophys. J. Suppl. 2010, 191, 160-178. [CrossRef]

99. Vacca, W.D.; Robert, C.; Leitherer, C.; Conti, P.S. The stellar content of 30 doradus derived from spatially integrated ultraviolet spectra: A test of spectral synthesis models. Astrophys. J. 1995, 444, 647-662. [CrossRef]

100. Smith, L.J.; Crowther, P.A.; Calzetti, D.; Sidoli, F. The Very Massive Star Content of the Nuclear Star Clusters in NGC 5253. Astrophys. J. 2016, 823, 38. [CrossRef]

101. Levesque, E.M.; Leitherer, C.; Ekstrom, S.; Meynet, G.; Schaerer, D. The Effects of Stellar Rotation. I. Impact on the Ionizing Spectra and Integrated Properties of Stellar Populations. Astrophys. J. 2012, 751, 67. [CrossRef]

102. Eldridge, J.J.; Stanway, E.R.; Xiao, L.; McClelland, L.A.S.; Taylor, G.; Ng, M.; Greis, S.M.L.; Bray, J.C. Binary Population and Spectral Synthesis Version 2.1: Construction, Observational Verification, and New Results. Pub. Astron. Soc. Austr. 2017, 34, e058. [CrossRef]

103. Moffat, A.F.J.; Corcoran, M.F.; Stevens, I.R.; Skalkowski, G.; Marchenko, S.V.; Mücke, A.; Ptak, A.; Koribalski, B.S.; Brenneman, L.; Mushotzky, R.; et al. Galactic Starburst NGC 3603 from X-Rays to Radio. Astrophys. J. 2002, 573, 191-198. [CrossRef]

104. Melena, N.W.; Massey, P.; Morrell, N.I.; Zangari, A.M. The Massive Star Content of NGC 3603. Astron. J. 2008, 135, 878-891. [CrossRef]

105. Ramachandran, V.; Hamann, W.R.; Oskinova, L.M.; Gallagher, J.S.; Hainich, R.; Shenar, T.; Sander, A.A.C.; Todt, H.; Fulmer, L. Testing massive star evolution, star formation history, and feedback at low metallicity. Spectroscopic analysis of OB stars in the SMC Wing. Astron. Astrophys. 2019, 625, A104. [CrossRef]

106. Johnson, T.L.; Rigby, J.R.; Sharon, K.; Gladders, M.D.; Florian, M.; Bayliss, M.B.; Wuyts, E.; Whitaker, K.E.; Livermore, R.; Murray, K.T. Star Formation at $\mathrm{z}=2.481$ in the Lensed Galaxy SDSS J1110+6459: Star Formation Down to 30 pc Scales. Astrophys. J. Lett. 2017, 843, L21. [CrossRef] [PubMed]

107. Baldwin, J.A.; Phillips, M.M.; Terlevich, R. Classification parameters for the emission-line spectra of extragalactic objects. Pub. Astron. Soc. Pac. 1981, 93, 5-19. [CrossRef]

108. Micheva, G.; Oey, M.S.; Jaskot, A.E.; James, B.L. Mrk 71/NGC 2366: The Nearest Green Pea Analog. Astrophys. J. 2017, 845, 165. [CrossRef] 
109. Steidel, C.C.; Rudie, G.C.; Strom, A.L.; Pettini, M.; Reddy, N.A.; Shapley, A.E.; Trainor, R.F.; Erb, D.K.; Turner, M.L.; Konidaris, N.P.; et al. Strong Nebular Line Ratios in the Spectra of z 2-3 Star Forming Galaxies: First Results from KBSS-MOSFIRE. Astrophys. J. 2014, 795, 165. [CrossRef]

110. Izotov, Y.I.; Orlitová, I.; Schaerer, D.; Thuan, T.X.; Verhamme, A.; Guseva, N.G.; Worseck, G. Eight per cent leakage of Lyman continuum photons from a compact, star-forming dwarf galaxy. Nature 2016, 529, 178-180. [CrossRef]

111. Izotov, Y.I.; Schaerer, D.; Thuan, T.X.; Worseck, G.; Guseva, N.G.; Orlitová, I.; Verhamme, A. Detection of high Lyman continuum leakage from four low-redshift compact star-forming galaxies. Mon. Not. R. Astron. Soc. 2016, 461, 3683-3701. [CrossRef]

112. Steidel, C.C.; Strom, A.L.; Pettini, M.; Rudie, G.C.; Reddy, N.A.; Trainor, R.F. Reconciling the Stellar and Nebular Spectra of High-redshift Galaxies. Astrophys. J. 2016, 826, 159. [CrossRef]

113. Kennicutt, R.C., Jr.; Lee, J.C.; Funes, J.G.; Sakai, S.; Akiyama, S. An H $\alpha$ Imaging Survey of Galaxies in the Local 11 Mpc Volume. Astrophys. J. Suppl. 2008, 178, 247-279. [CrossRef]

114. van Zee, L.; Skillman, E.D.; Haynes, M.P. Oxygen and Nitrogen in Leo A and GR 8. Astrophys. J. 2006, 637, 269-282. [CrossRef]

115. Saviane, I.; Rizzi, L.; Held, E.V.; Bresolin, F.; Momany, Y. New abundance measurements in UKS 1927-177, a very metal-poor galaxy in the Local Group. Astron. Astrophys. 2002, 390, 59-64. [CrossRef]

116. Garcia, M.; Herrero, A.; Najarro, F.; Camacho, I.; Lorenzo, M. Ongoing star formation at the outskirts of Sextans A: Spectroscopic detection of early O-type stars. Mon. Not. R. Astron. Soc. 2019, 484, 422-430. [CrossRef]

117. Walborn, N.R.; Nichols-Bohlin, J.; Panek, R.J. International Ultraviolet Explorer Atlas of O-type Spectra from 1200 to $1900 \AA$; Technical Report NASA-RP-1155, NAS 1.61:1155; NASA Goddard Space Flight Center: Greenbelt, MD, USA, 1 December 1985; p. 56.

(C) 2019 by the authors. Licensee MDPI, Basel, Switzerland. This article is an open access article distributed under the terms and conditions of the Creative Commons Attribution (CC BY) license (http:/ / creativecommons.org/licenses/by/4.0/). 